\title{
EL PDC: BASES ELECTORALES, DETERMINANTES DE ADHESIÓN E IMPACTO EN LAS VOTACIONES DE R. LAGOS Y M. BACHELET ${ }^{1}$
}

\author{
Mauricio Morales \\ y Antonio Poveda
}

\begin{abstract}
En este estudio se concluye que religión y escolaridad son las variables que mejor explican el desempeño electoral del Partido Demócrata Cristiano (PDC) y su adhesión en las encuestas. Al comparar los datos de 1990 y 2005, la variable más estable es el nivel de educación de los encuestados. Se constata escasa disposición de los "universitarios" para adherir al partido y, a nivel comunal, a mayor escolaridad promedio, menor votación por el PDC. En cuanto a la definición católica de los encuestados, se encuentra que su mayor impacto en la adhesión al PDC ocurre en 1990, situación que se
\end{abstract}

Mauricio Morales Quiroga. Periodista, cientista político y magíster en ciencias sociales por la FLACSO-México. Profesor e investigador del Instituto de Ciencias Sociales de la Universidad Diego Portales y estudiante del Doctorado en Ciencia Política de la Pontificia Universidad Católica de Chile. E-mail: mauricio.morales@prof.udp.cl.

Antonio Poveda Ribot. Bachiller en Ciencias Sociales y alumno de quinto año de la carrera de Ciencia Política, Universidad Diego Portales. E-mail: antonio.poveda@al.udp.cl

${ }^{1}$ Este trabajo recibió financiamiento del Proyecto Fondecyt 1060479 (Evolución histórica y determinantes sociales, étnicos, culturales y coyunturales del comportamiento electoral de los chilenos, 1989-2005) y del Fondo Semilla Número 160325018, financiado por la Universidad Diego Portales. Agradecemos los comentarios de los miembros del Observatorio Electoral del Instituto de Ciencias Sociales de la Universidad Diego Portales y del evaluador anónimo de Estudios Públicos que revisó el manuscrito. Como siempre, la responsabilidad por los contenidos del artículo recaen exclusivamente en los autores.

Estudios Públicos, 107 (invierno 2007). 
mantiene durante la década y que, hacia 2005, disminuye si se consideran los datos de las encuestas, mas no a nivel de resultados electorales por comuna. A continuación se observa un menor respaldo del PDC a las candidaturas de Ricardo Lagos y Michelle Bachelet en comparación con el resto de los partidos de la Concertación, lo que se corrobora tanto a nivel comunal como individual. Finalmente se advierte que a pesar de la baja electoral del PDC, aún persisten algunos patrones en su votación, y que su fuerza dentro de la Concertación parece indispensable para el éxito electoral del bloque.

\section{Presentación}

$\mathrm{D}$ esde 1997 el PDC ha evidenciado una baja electoral que, particularmente en 2001 y 2005, provocó una significativa disminución en el tamaño de sus bancadas parlamentarias. Varias razones han sido esgrimidas para dar cuenta de este declive. Según Huneeus (2002, 2002a y 2003), la caída del PDC se explica por su desgaste natural al mando del gobierno en dos períodos consecutivos, la falta de renovación de sus elites y el enfrentamiento en una competencia bilateral con el Partido por la Democracia (PPD) y la Unión Demócrata Independiente (UDI)². Además, sugiere que el PDC ha tenido severos problemas de institucionalización en los 90 asociados a su falta de adaptación, y que no fue capaz de resolver plenamente el problema sucesorio luego del gobierno de Patricio Aylwin. La opinión de Arriagada (2001 y 2005), en tanto, profundiza en la obsolescencia programática del PDC, los problemas de liderazgo que muchas veces conducen a las directivas a actuar en beneficio propio y no del partido, y la falta de un criterio eficiente para la conformación de las listas parlamentarias. A esto se adiciona la visión de Walker (1999: 132), destacando la longevidad del partido, las lógicas burocráticas y la existencia de grupos de poder. Desde una óptica estrictamente electoral, Navia (2002) explica la caída del PDC conside-

${ }^{2}$ La competencia bilateral, expresada en la intención de dos partidos por "adquirir" declaradamente las históricas bases electorales de otro, se explica por la pública intención de la UDI para atraer a los votantes cautivos del PDC, y por los intentos del PPD para posicionarse como un partido de centro y "paraguas" dentro de la Concertación (ver Huneeus, 2003). Incluso, esto se ha evidenciado en el mismo análisis electoral. Las derrotas de los candidatos incumbentes del PDC han sido, en la mayoría de los casos, producidas por representantes de la UDI y del PPD. 
rando el desequilibrio de su votación regional ${ }^{3}$ y, además, por la pérdida de adhesión entre las mujeres, grupo que constituía una de sus principales bases de apoyo.

Para contribuir a la discusión de esos diagnósticos y visiones, en este trabajo intentamos identificar empíricamente los determinantes sociodemográficos y socioeconómicos de la votación democratacristiana. Por otro lado, también nos proponemos medir el impacto de la votación DC en el desempeño electoral de Ricardo Lagos y Michelle Bachelet; es decir, evaluar su peso relativo en las candidaturas presidenciales de la coalición (Concertación) que no fueron democratacristianas. "La votación DC” constituye así la variable dependiente en el primer análisis y la variable independiente en el segundo. De acuerdo a esto, nos formulamos las siguientes preguntas de investigación: ¿¿cuáles son las características de la base electoral del PDC, considerando su rendimiento a nivel comunal y su adhesión en las encuestas?, ¿existen patrones de votación asociados a variables socioeconómicas y sociodemográficas?, ¿qué tan importante es la votación DC para los candidatos presidenciales de la Concertación que no fueron militantes del partido? Estas preguntas se enmarcan en el estudio de los partidos en el electorado, sin responder a su presencia en el Congreso ni en la sociedad civil, como tampoco al análisis de su organización (Montes et al., 2000).

Para nuestro estudio nos basamos en resultados electorales a nivel comunal, así como en datos del Sistema Nacional de Indicadores Municipales (SINIM), del Censo 1992 y 2002, de la Encuesta de Caracterización Sociodemográfica (CASEN) y de las encuestas de opinión del Centro de Estudios Públicos (CEP).

Metodológicamente, trabajamos con dos unidades de análisis: comunas e individuos. Cada una de estas unidades tiene sus propias bondades y limitaciones. A nivel comunal contamos con los resultados reales de la elección, y por otro lado, es la unidad de análisis electoral más pequeña en la cual podemos utilizar, por ejemplo, datos a nivel socioeconómico y sociodemográfico. Esos datos agregados, sin embargo, no nos permiten llegar al votante. Con las encuestas, en tanto, tenemos la ventaja de analizar individuos, aun cuando no haya certeza respecto a cómo realmente votaron. Así, la utilización de ambas unidades de análisis nos permite resolver algunos problemas relativos a la "falacia ecológica", adquiriendo los resultados mayor consistencia y solidez.

${ }^{3}$ Según Navia (2002), este desequilibrio regional se expresa básicamente por las constantes caídas del PDC en los sectores urbanos más poblados, particularmente en las regiones Quinta y Metropolitana. 
El trabajo queda constituido de la siguiente manera: en la segunda sección describimos el rol del PDC en el sistema de partidos chileno y su desempeño electoral considerando los comicios de diputados y municipales. La tercera sección está dedicada al análisis de las bases electorales del partido acudiendo a variables socioeconómicas y sociodemográficas. En cuarto lugar se estudia el impacto de la votación por el PDC sobre las candidaturas presidenciales de Ricardo Lagos y Michelle Bachelet. La última parte del trabajo está destinada a la exposición de las principales conclusiones.

\section{El PDC en el sistema de partidos}

Durante los 80 la expresión política de la oposición a Pinochet fue la Concertación y dentro de ella el liderazgo fue ejercido por el PDC (Angell, 2003; Drake y Jaksic, 1999; Walker, 1999) ${ }^{4}$. En 1990 logró la Presidencia de la República con Patricio Aylwin y en 1994 con Eduardo Frei Ruiz-Tagle. Desde la reinauguración democrática el sistema de partidos, definido como de multipartidismo moderado, se ha estructurado sobre la base de dos grandes coaliciones, la Concertación y la Alianza (Siavelis, 1997; Tironi y Agüero, 1999; Valenzuela, 1999; Torcal y Mainwaring, 2003). En este contexto, el PDC no sólo colaboró activamente en la derrota del general Pinochet para el plebiscito de 1988, sino que además se constituyó como el pilar de la coalición de gobierno durante los primeros diez años de democracia, contribuyendo al afianzamiento de las instituciones políticas en el marco de una sociedad emergente (Walker, 1999; Huneeus, 2003). Durante los 90, en tanto, el PDC fue el partido más votado del país, aunque perdió cierto protagonismo para las parlamentarias de 1997. Luego, con su candidato Andrés Zaldívar, sufrió una estrepitosa derrota en la primaria presidencial de la Concertación en 1999 a manos de Ricardo Lagos. Finalmente, en las parlamentarias de 2001 y 2005 nuevamente vio disminuidas sus bancadas de diputados y senadores.

Si bien es innegable que el PDC ha bajado su votación desde las municipales de 1996, en las de 2004 retomó el cetro del partido más grande de Chile, echando por tierra los supuestos sobre su extinción. No obstante, existe una suerte de tradición que anuncia la inexorable caída y desaparición

${ }^{4}$ Según Walker (1999: 137): “en ese contexto, la Democracia Cristiana no sólo supo asumir una clara postura en torno a la cuestión de los derechos humanos, sino que, especialmente desde comienzos de la década de 1980, se transformó en una gran fuerza articuladora de todos los que postulaban una salida pacífica a la dictadura, en la perspectiva de la consolidación de una democracia estable”. 
del PDC no sólo en Chile, sino que también en Europa (Fleet, 1985; Hanley, 1994; Kalyvas, 1996). A pesar de haber disminuido nuevamente su bancada de diputados en 2005, el PDC sigue siendo un partido relevante, más aún si consideramos el incremento de su número de alcaldes en los últimos comicios locales de 2004. Esto bien podría responder a la hipótesis de Walker (1999: 81), respaldada por Navarrete (2003: 153), respecto a que el partido está experimentando un ciclo electoral y no un declive terminal de su votación. A esto se añade que parte importante de la población, conforme a las encuestas de opinión, aún se identifica con posturas de centro ${ }^{5}$. Por último, el PDC es fundamental para la Concertación por el caudal electoral que aporta al bloque, como también por la provisión de cuadros técnicos destinados a la administración pública (Huneeus, 2003).

Frente a lo postulado tanto por Duverger (1951), en cuanto a que el centro no existe en política (puede haber un partido de centro, pero no hay una tendencia de centro), como por Sartori (1992), respecto a que el centro no es más que una convergencia negativa (es decir, una suma de exclusiones más que una agencia positiva de instigación), Scully (1992) ha respondido que una tendencia de centro no sólo puede existir, sino que, en el caso de Chile, ha existido siempre y sigue existiendo. Agrega que el centro ha permanecido y, lejos de tener poca importancia, ha sido protagonista principal del sistema de partidos chileno. Sostiene, además, que el PDC ha sido fundamental para la mantención de un sistema político estable, efectivo y con altos índices de gobernabilidad en Chile. El centro, argumenta Scully (1992), mantiene cohesionado el sistema de partidos dado su papel de mediador y, además, absorbe las perturbaciones del sistema que emanan de los polos ideológicos. Así, el PDC, como partido de centro, habría resistido incluso los efectos del sistema binominal, que más que tender al votante mediano incentivaría la captación de electores más extremos, ya sean de derecha o de izquierda (véanse al respecto, Magar et al., 1998; y Navia, 2005). En este sentido el sistema de partidos chileno mantendría su configuración en los tradicionales "tercios" — derecha, centro, izquierda— (véanse

${ }^{5}$ Por ejemplo, de acuerdo con los datos de la última encuesta del Instituto de Ciencias Sociales de la Universidad Diego Portales, el promedio que se alcanza en la escala política de 1 (muy de izquierda) a 10 (muy de derecha) es de 5,21, excluyendo a aquellos que en esta pregunta no se inclinan por ninguna de las alternativas. Cuando se consulta por la ubicación de los partidos en este mismo eje, el PDC logra una media de 5,23, siendo el partido más cercano a las posturas de centro que, en el total nacional y sumando los valores 5 y 6, sobrepasa el 50\%. Esto es consistente con los resultados que arroja el trabajo de Huneeus (2003), donde se evidencia que el PDC es visto, efectivamente, como un genuino partido de centro. 
Garrido y Navia, 2005; Valenzuela, 1995 y 1999), a pesar del sistema electoral, el que no ha sido capaz de cumplir su principal objetivo, esto es, reducir la fragmentación partidaria (véase Cabezas y Navia, 2005).

\section{El PDC y su desempeño electoral}

La DC llegó al poder en 1964. El 4 de septiembre de aquel año, con su líder Eduardo Frei Montalva, y apoyado por otros grupos políticos y numerosos independientes, obtuvo una resonante victoria en las elecciones presidenciales. Frei Montalva se convirtió en el primer presidente del PDC en la historia de Chile, obteniendo el más alto porcentaje de sufragios (56,09\%) recibidos por un candidato en la historia constitucional democrática del país iniciada en 1932 y vigente hasta 1973. La experiencia comparada, además, indicaba el desarrollo de los partidos demócratas cristianos en Europa, particularmente en Alemania e Italia (Fogarty, 1958).

Frei Montalva gobernó entre 1964 y 1970 con el lema "Revolución en libertad”. Uno de sus principales legados fue entregar nuevas oportunidades de participación en los asuntos públicos a la ciudadanía. Ejemplo de ello fue la promoción popular y la sindicalización campesina y de los trabajadores industriales, estableciendo el paso de una política oligárquica a otra eminentemente social (Arriagada, 1986; Garretón, 1990; Ortega Frei, 1992; Boeninger, 1997; Walker, 1999).

El triunfo del socialista Salvador Allende en 1970 profundizó el proceso socio-político de un liderazgo horizontal iniciado por el gobierno de Frei Montalva. Sin embargo, la profunda crisis económica, social y política que afectaba al país llevó a parte importante de la DC a no condenar oficialmente el golpe militar de 1973 (Arriagada, 1974). Algunos, como Arturo Valenzuela (2003), incluso la consideran como la gran responsable de la crisis de este gobierno que derivó en el golpe de Estado, producto de su incapacidad para transformarse en el imán del sistema político y convertirse en un punto de fuga de los partidos.

Durante el régimen autoritario, como todas las agrupaciones políticas, la DC permaneció en la clandestinidad. En la década de los 80 publicó las bases institucionales de una reconciliación nacional y de una transición a una democracia estable. A pesar del rechazo de Pinochet al diálogo, hechos significativos, como la visita de Juan Pablo II, establecieron la antesala de lo que sería la posterior derrota de Pinochet en el plebiscito de 1988 y de la derecha en las elecciones presidenciales de 1989 (Boeninger, 1997; Cavallo et al., 1999; Otano, 1995). 
La DC encabezó el camino del reencuentro de la sociedad con la democracia, y las caras de los primeros años de la Concertación eran principalmente figuras del partido. Por ejemplo, Patricio Aylwin, Presidente de la República (1990-1994), y Gabriel Valdés, Presidente del Senado. La DC durante los 90, gracias a su notable desempeño electoral, se convirtió en el partido más importante de la expresión política de la época: la Concertación (Cavallo, 1998). Este hecho no es menor considerando los desafíos propios de la transición y, aún más importante, los retos que implica la redemocratización de las sociedades luego de los regímenes autoritarios (Dix, 1992). Además, el reestreno de los partidos políticos en Chile no fue un proceso rápido ni mucho menos expedito. Las trabas del régimen autoritario y el miedo constante a represalias fueron algunos factores que influyeron en la búsqueda de un partido de centro capaz de liderar el proceso. Acá, el PDC jugó un rol central donde nuevamente los partidos asumían un rol clave dentro de las nuevas reglas de la democracia (Morlino, 1995; Lipset, 2000) ${ }^{6}$.

El predominio del PDC fue incuestionable a comienzos de la década de los 90. Respecto a los comicios de diputados en las elecciones de 1989 (ver Figura N ${ }^{\circ}$ 1), la Concertación obtuvo 3.499.713 votos y la DC 1.776.347, alcanzando el 25,99\% del total nacional. En las elecciones de 1993, la votación de la Concertación fue de 3.733.276 votos, obteniendo la DC un 27,12\% con 1.827.373 sufragios. Para el año 1997, tanto la votación de la Concertación como la de la DC bajaron respecto a la parlamentaria anterior. La Concertación obtuvo 2.927.692 votos y la DC 1.331.745, alcanzando el 22,98\%. La votación de la coalición centro-izquierda se mantuvo en la elección de diputados 2001, donde la Concertación obtuvo 2.942 .989 votos y la DC 1.162.210, alcanzando el 18,92\%, mientras que en la última elección de diputados del año 2005 aumentó la votación oficialista y del PDC: la Concertación obtuvo 3.374.865 votos y la DC 1.354.631, logrando el 20,78\% y ubicándose menos de dos puntos más abajo que la UDI.

La Figura $\mathrm{N}^{\circ} 1$ muestra, además, los beneficios que obtuvo el PDC con el sistema binominal en las elecciones de 1993 y 1997. Con menos votos “compró” más escaños, aprovechando así los vicios de la desproporcionalidad. Pero en las elecciones subsiguientes el escenario pareció ser el opuesto. Si bien el 2001 el PDC casi igualó su porcentaje de votos con su porcentaje de escaños, para el 2005 la situación fue distinta. Con un 20,78\% de los votos, el PDC accedió a 21 escaños, lo que representó un 17,5\% de la

${ }^{6}$ Según Morlino (1995), los partidos políticos son fundamentales para el proceso de consolidación democrática y para el fortalecimiento del orden pluralista. A esto se adiciona, siguiendo a Lipset (2000), el rol de los partidos de oposición para fiscalizar las acciones del gobierno en el marco de la democracia, por lo que los partidos son considerados como "indispensables". 
FIGURA N $N^{\circ}$ 1: $\quad$ VOTOS Y ESCAÑOS DEL PDC EN LAS ELECCIONES DE DIPUTADOS 1989-2005, EN PORCENTAJE

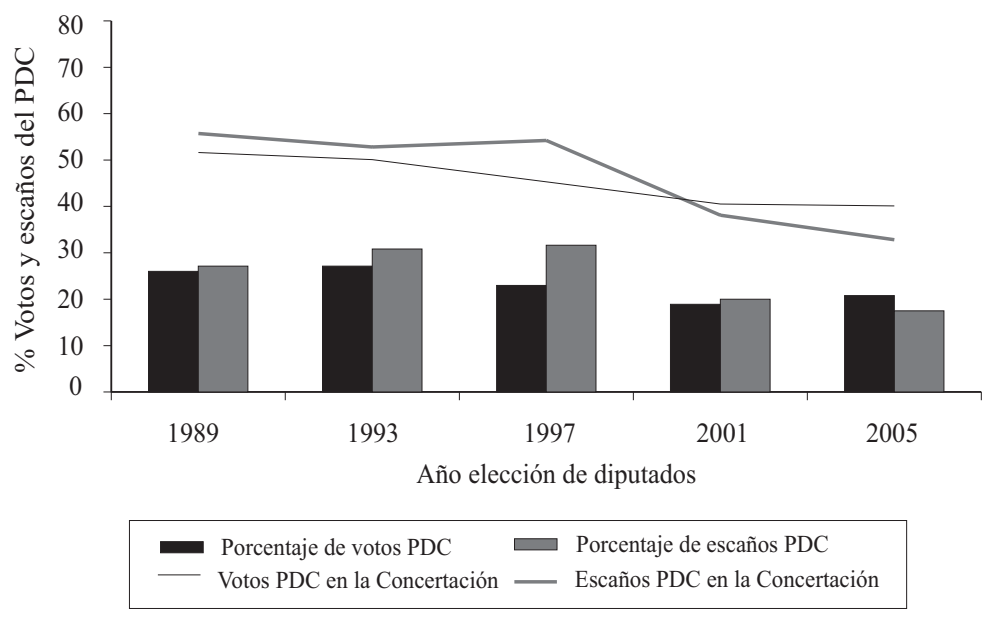

Fuente: Elaboración propia con datos de www.elecciones.gov.cl

Cámara. Luego, la participación del PDC dentro de la Concertación ha venido en franca caída, particularmente en porcentaje de votos. Recién el 2005 se presentó una leve recuperación, pero, paralelamente, empeoró el porcentaje de escaños del PDC dentro de la Concertación. De esta forma, la detención de la caída en votos no ha ido de la mano con el mismo resultado en escaños.

Otra información relevante corresponde al número de distritos donde el PDC ha sido capaz de sobrepasar el Margen Absoluto de Seguridad Electoral (MAS) que tiene el sistema binominal. Éste corresponde a un 33,3\% periódico más un voto. En 1989, 18 de sus candidatos sobrepasaron este margen, al igual que en 1993. Luego, en 1997 y 2005 sólo diez diputados lograron este objetivo, mientras que en 2001 sólo seis. A pesar de esto, el PDC obtuvo menos diputados el 2005 que el 2001. Tal antecedente nos podría estar informando acerca de determinados distritos donde será difícil observar una derrota del partido. Éstos son los casos de los distritos 4, 6, 8, 34, 35, 36, 38, 48, 55, 58. Ninguno de ellos corresponde a la Región Metropolitana, que es la más poblada del país representando alrededor del $40 \%$ de los electores. Además se advierte, al menos para 1993, que la votación del PDC no tenía gran variabilidad. De hecho, ninguno de sus candidatos bajó del 19\% de la votación. En cambio, en las elecciones de 2005 hubo 
seis candidatos que no fueron capaces de superar la barrera del 10\%. Incluyéndolos, fueron 22 los candidatos del PDC que no llegaron al 20\%. Esto nos retrata de buena forma el proceso de desgaste del partido y los problemas de reclutamiento para seleccionar candidatos medianamente competitivos.

En el ámbito municipal (ver Figura № 2), en 1992 la Concertación obtuvo 3.417.154 votos y la DC aportó con 1.854.679, alcanzando el 28,93\% de la votación nacional. En las elecciones municipales de 1996, la Concertación elevó su votación municipal a 3.536.842 y la DC aportó 1.640.108 votos, obteniendo el 26,03\%. Para las municipales de 2000, la Concertación logró 3.396.274 y la DC 1.408.445, representando el 21,62\%. En las elecciones de alcaldes de 2004, la Concertación alcanzó 2.827.514 votos y la DC 1.382.185, logrando el 21,90\%, mientras que en la de concejales obtuvo el 20,3\%. En este sentido, y respecto al número de alcaldes y concejales DC para estas elecciones, cabe señalar que para las de 1992 la DC obtuvo 144,5 alcaldías (43,26\%) y 498,5 escaños en concejales (28,52\%) ${ }^{7}$. En el año 1996 eligió 104 alcaldes (30,50\%) y 476 concejales (26,61\%), mientras que para las eleccio-

FIGURA N ${ }^{\circ}$ 2: $\quad$ VOTOS Y ALCALDÍAS DEL PDC EN LAS MUNICIPALES 1992-2004, EN PORCENTAJES

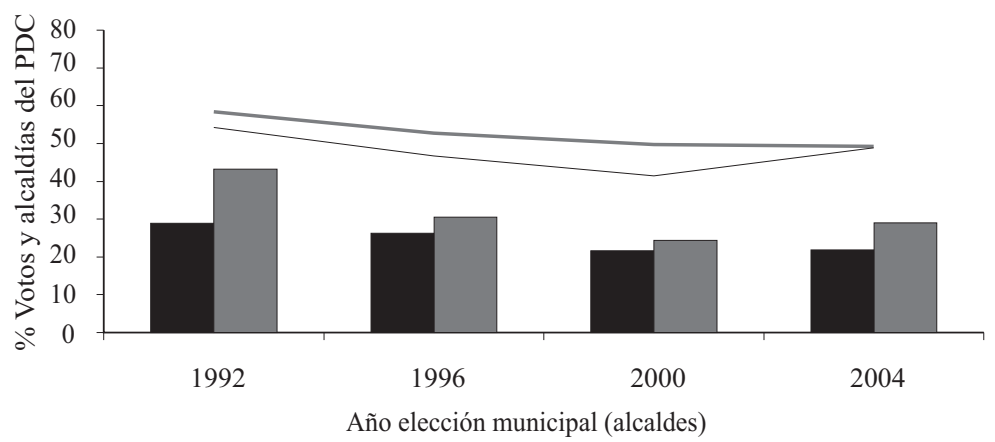

Fuente: Elaboración propia con datos de www.elecciones.gov.cl

${ }^{7}$ En 1992 las cifras fraccionadas en el número de alcaldes y concejales se deben a que algunos mandatos fueron divididos en mitades, siendo asignada la alcaldía por dos años a un concejal y por los dos siguientes a otro (Mardones, 2006: 22). 
nes de 2000, el PDC alcanzó 83 alcaldías (24,34\%) y 419 concejales (23,50\%). Finalmente, para las elecciones de 2004, la DC logró 100 alcaldías (28,99\%) y 455 concejales (21,22\%) (Mardones, 2006: 20-21).

De la Figura $N^{\circ} 2$ se desprende una interpretación relevante. El PDC en todas las elecciones municipales ha obtenido más porcentaje de alcaldías que porcentaje de votos. Esto fue más evidente en 1992, elección en que el PDC se alzó con un rotundo triunfo logrando el 43,26\% de los alcaldes. De ahí en adelante el desempeño electoral del partido ha sido estable. No se observan caídas de la magnitud de las elecciones parlamentarias y, si bien ha perdido participación dentro de la Concertación, en las elecciones de concejales 2004 sólo disminuyó levemente su votación con respecto al 2000. En cambio, en las elecciones de alcaldes, que son las que figuran en el gráfico, el PDC incrementó notoriamente su porcentaje de alcaldías, remontando así la baja que sufrió en las municipales del 2000. Esto no sólo se debió a un aumento de su votación, sino que también a la negociación favorable que logró en la mesa de la Concertación. Mediante la cesión al PPD de la candidatura a alcalde por la comuna de Santiago, el PDC obtuvo el $47 \%$ de las candidaturas a concejales y 149 cupos (43,2\%) para candidatos a alcalde.

Si bien los resultados electorales recién descritos dan cuenta del éxito electoral de la DC durante los 90, puesto que obtuvo la primera mayoría en gran parte de las elecciones, en las parlamentarias de 2001 dejó de ser el partido más votado dando paso a la UDI, categoría que había mantenido desde las elecciones municipales de 1963. Sin embargo, como enunciamos, los resultados de las elecciones municipales de 2004 muestran que entre la municipal y la parlamentaria de 2001 la DC subió 1,35 puntos, recuperando así su condición de principal partido al superar a la UDI. Luego, en las parlamentarias 2005, y sin llevar candidato presidencial debido al retiro de Soledad Alvear de la primaria interna de la Concertación, el PDC obtuvo 1.354.631 votos, representando el 20,78\%. Su bancada bajó de 24 a 21 escaños en comparación con la legislatura anterior, mientras que en el Senado también se produjo una caída, esta vez de 11 a 6 escaños.

\section{Identificando la base electoral del PDC}

En esta sección investigamos hasta qué grado la votación DC responde a características socioeconómicas y sociodemográficas de la población. Para ello hemos construido una base de datos con los resultados electorales por comuna para los comicios de diputados de 1989 y 2005, en 
conjunto con información socioeconómica y sociodemográfica de las comunas tomada del Sistema Nacional de Indicadores Municipales (SINIM), Censo 1992 y 2002, y Encuesta de Caracterización Sociodemográfica (CASEN).

El objetivo consiste en evaluar si existen variables socioeconómicas y sociodemográficas que expliquen la votación por el PDC en la elecciones de diputados de 1989 y de 2005. Estudios como los de Rosenstone y Hansen (2002) y Niemi y Weisberg (2001) indican que variables de este tipo se relacionarían con las preferencias políticas. La Tabla $\mathrm{N}^{\circ} 1$ muestra regresiones de mínimos cuadrados que dan cuenta de estas relaciones.

Es importante señalar que en la Tabla $\mathrm{N}^{\circ} 1$, al igual que en las siguientes, presentamos tanto coeficientes ponderados como no ponderados. La ponderación la realizamos con los votos emitidos por comuna, a fin de calcular el peso relativo que tiene cada una de ellas en el total nacional. También se pueden tomar otras medidas de ponderación, como el número de inscritos o el total de votos válidamente emitidos, aunque con las tres medidas los coeficientes ponderados tienen un valor muy similar. Al efectuar la ponderación prácticamente descomponemos la unidad de análisis original que es la comuna, calculando su peso relativo dentro del total, toda vez que en Chile la varianza poblacional entre las comunas es altísima. Por ejemplo, en Tortel, en 2004, votaron 284 personas, mientras que en Puente Alto lo hicieron 108.738.

Hemos construido cuatro modelos por año considerando los eventuales problemas de colinealidad que se darían entre ruralidad, pobreza y escolaridad promedio. Aunque los coeficientes de tolerancia son relativamente aceptables, optamos por eliminar algunas de estas variables en los modelos 2, 3 y 4 a fin de no distorsionar los resultados ${ }^{8}$ (véanse los modelos en la Tabla $\mathrm{N}^{\circ} 1$ ).

Las interpretaciones para la primera observación, es decir, 1989, son altamente cuestionables. Esto, debido al bajo número de casos donde encontramos datos electorales en conjunto con información sociodemográfica. Los $\mathrm{R}^{2}$ son bajísimos y no nos entregan mayores noticias sobre el desempeño electoral del PDC de acuerdo con las características comunales. Además, ninguna de las variables es significativa en los datos sin ponderar.

${ }^{8}$ En el primer modelo de 1989 los valores de tolerancia son de 0,669 para porcentaje de católicos, 0,411 para escolaridad promedio, 0,935 para porcentaje de población rural y 0,328 para porcentaje de pobres. En 2005 y también en el primer modelo, los valores de tolerancia son de 0,736 para porcentaje de católicos, 0,283 para escolaridad promedio, 0,381 para porcentaje de población rural y 0,488 para porcentaje de pobres. 


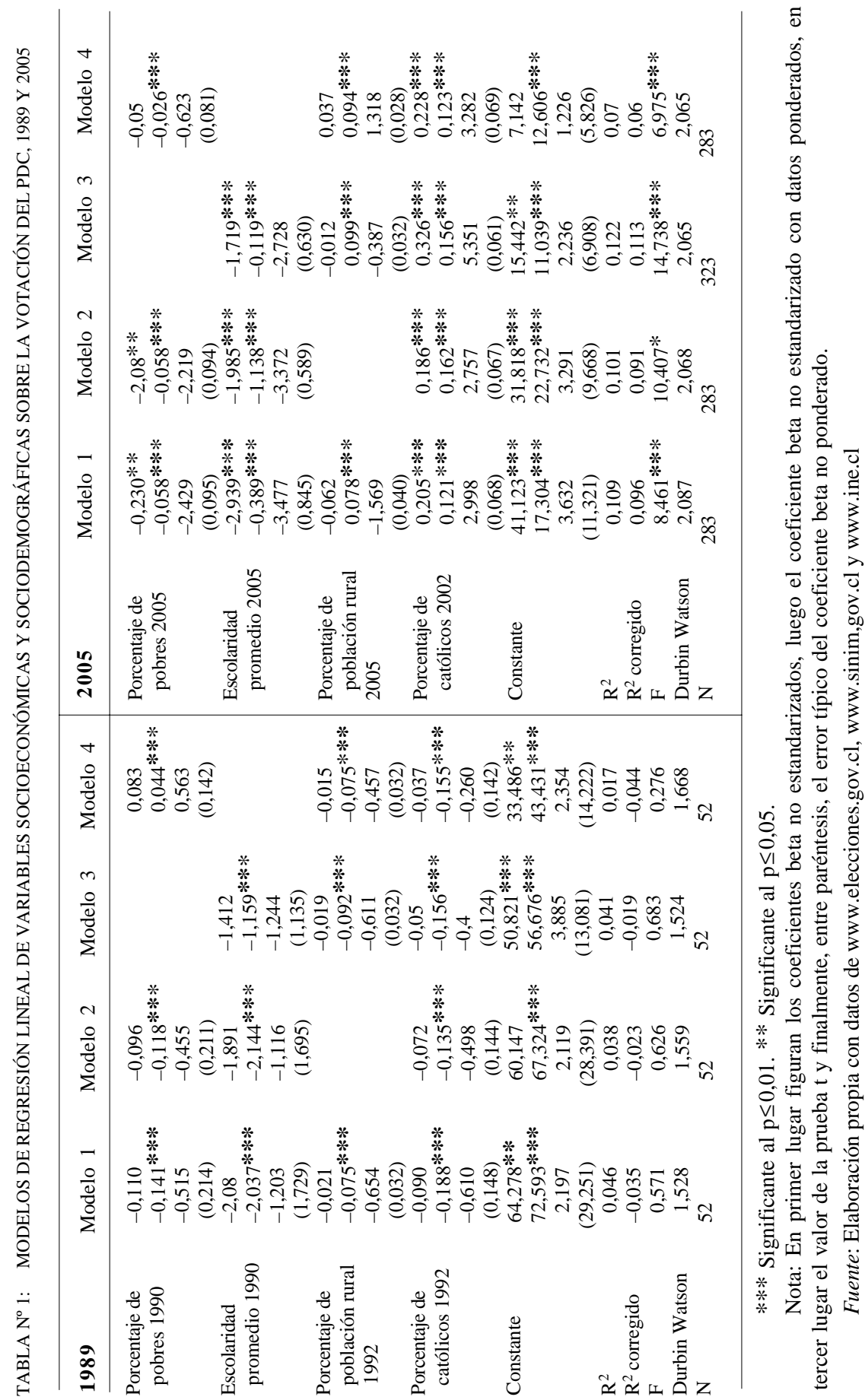


Lo anterior cambia para 2005. Como vemos, la única variable que mantiene significancia estadística en todos los modelos, ponderados y no ponderados, es porcentaje de católicos y escolaridad promedio. Manteniendo el resto de las variables constantes, el incremento del porcentaje de católicos impacta positivamente en la votación por el PDC, sucediendo lo contrario con la escolaridad promedio. Al estar presentes con significancia estadística en todos los modelos, estas dos variables se convierten en las más relevantes.

Sin embargo, las capacidades explicativas de todos los modelos son bajas. Se hace inapropiado ingresar más variables de orden socioeconómico y sociodemográfico a los modelos por los problemas de colinealidad que existen entre ellas. Esto provocaría un incremento de los $\mathrm{R}^{2}$ pero sólo de manera artificial. Indudablemente que, por ejemplo, el ingreso de variables asociadas con sindicalización podría ayudarnos a explicar de mejor forma la votación del PDC, pero son datos de los que no disponemos a nivel comunal.

Si bien el PDC se define como partido no confesional, es llamativo el impacto que sobre su votación genera el porcentaje de católicos (ver Figura $\mathrm{N}^{\circ} 3$ ). De hecho, el coeficiente de correlación entre ambas variables es de 0,298 y altamente significativo (***). El mismo coeficiente es negativo y significativo para el PS y para el PPD, mientras que con la votación de los partidos de derecha no existe relación estadísticamente significativa. Luego, de acuerdo con la población supuestamente más practicante, que es la evangélica, el coeficiente de correlación entre el PDC y este grupo es de

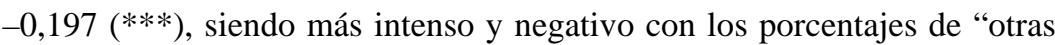
religiones” y de "ninguna religión” que alcanzan valores de -0,293 y -0,286, ambos con alta significancia estadística (***).

Esta correlación, con datos ponderados, baja a un $0,181(* * *)$. Por tanto, es muy probable que en los datos sin ponderar se haya sobre-estimado el efecto de las comunas más pequeñas. Si bien puede parecer extraño que para 1989 la relación entre el desempeño electoral del PDC y el porcentaje de católicos sea inexistente y negativa cuando se ponderan los casos, esto se debe, al menos, a tres factores. En primer lugar, en 1989 la votación del PDC fue más homogénea en comparación con 2005, que tuvo mayor nivel de dispersión considerando el desempeño comunal. Esto dificulta la captación de relaciones significativas con otras variables. Luego, para 2005 también se hace más variable la adscripción religiosa, apareciendo con fuerza el porcentaje de evangélicos y de "ninguna" religión. Por último, las elecciones de 1989 han sido catalogadas como "anormales" debido, entre otras cosas, a los distritos que cedió el PDC a otros partidos. 
FIGURA N ${ }^{\circ}$ 3: $\quad$ DIAGRAMA DE DISPERSIÓN ENTRE PORCENTAJE DE VOTOS DEL PDC EN DIPUTADOS 2005 Y PORCENTAJE DE CATÓLICOS POR COMUNA 2002

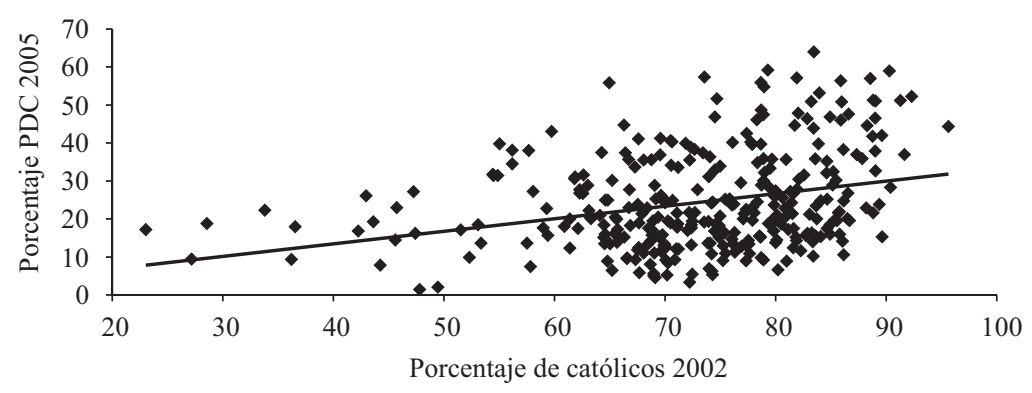

Fuente: Elaboración propia con datos de www.elecciones.gov.cl y www.ine.cl

Para aclarar todo lo anterior, y reconociendo los límites que tiene el análisis por comuna, pasamos a estudiar datos individuales. Presentamos aquí dos modelos de regresión logística binominales a partir de los resultados de encuestas de opinión (ver Tablas $\mathrm{N}^{\circ} 2$ y No 3 ), estableciendo ciertas similitudes con el trabajo de Ortega Frei (2003). Utilizamos variables de "largo plazo” que son recomendadas por el modelo sociológico (Lazarsfeld et al., 1944; Crewe, 1995) y la escuela de la identificación partidaria (Campbell et al., 1960; Jenings y Niemi, 1968; Converse, 1969; Richardson, 1991; Ventura, 2001). El supuesto fundamental sobre el que descansan argumentativamente estas escuelas es que el voto es un acto de afirmación. Mientras en el modelo sociológico se evidencian patrones de votación por clase social, en el de identificación partidaria se privilegian los aspectos sicológicos de adhesión a los partidos y donde la influencia familiar, en el proceso de socialización, es un claro determinante. Así, se postula que el votante adquiere la identificación con el partido desde el seno familiar, para luego consolidarse en los distintos grupos que integra (López, 2004: 286). Para este trabajo hemos seleccionado las siguientes variables: “sexo”, “edad”, “educación”, “nivel socioeconómico”, “ocupación” y “religión”. En este sentido, tratamos de encontrar evidencia respecto a la tendencia del PDC a recibir importante respaldo femenino (Navia, 2002), así como sobre su llegada a los estratos medios (Navarrete, 2003 y 2005) y mayor sintonía con sectores católicos, aunque se presente como un partido no confesional (Walker, 1999: 177). 
TABLA N ${ }^{\circ}$ 2: $\quad$ MODELO DE REGRESIÓN LOGÍSTICA PARA 1990. “ODDS RATIO” DE LOS DETERMINANTES DE ADHESIÓN AL PDC (1= PDC, 0= OTROS)

\begin{tabular}{|c|c|c|c|c|c|}
\hline & Modelo 1 & Modelo 2 & Modelo 3 & Modelo 4 & Modelo 5 \\
\hline Sexo (a) & 1,061 & 1,238 & 1,066 & 1,074 & 1,070 \\
\hline \multicolumn{6}{|l|}{ Edad (b) } \\
\hline $18-24$ & 1,372 & 0,803 & 1,042 & 1,310 & 1,062 \\
\hline $25-34$ & $2,005(+)$ & 1,102 & 1,480 & $2,498 * * *$ & $1,943 * *$ \\
\hline $35-44$ & 1,284 & 0,903 & 1,037 & 1,542 & 1,299 \\
\hline $45-54$ & 1,723 & 1,318 & 1,550 & 2,097 & $1,936 * *$ \\
\hline $55-64$ & 1,252 & 1,151 & 1,164 & $1,457 * * *$ & 1,377 \\
\hline \multicolumn{6}{|l|}{ NSE (c) } \\
\hline ABC1 & 2,463 & 0,962 & & 2,495 & \\
\hline C2 & $2,915(+)$ & 1,531 & & 2,698 & \\
\hline C3 & 2,475 & 1,944 & & 2,551 & \\
\hline $\mathrm{D}$ & 1,348 & 1,173 & & 1,400 & \\
\hline \multicolumn{6}{|l|}{ Educación (d) } \\
\hline Sin estudios & 1,698 & & 1,140 & 1,692 & 1,170 \\
\hline Básica incompleta & $4,265 * *=$ & & $3,328 * * *$ & $4,306 * * *$ & $3,420 * * *$ \\
\hline Básica completa & $2,523 * *=$ & & $2,016 * *$ & $2,612 * * *$ & $2,128 * * *$ \\
\hline Media incompleta & $3,117^{* *}$ & & $2,932 * * *$ & $3,181 * * *$ & $2,976 * * *$ \\
\hline Media completa & 1,129 & & 1,086 & 1,150 & 1,116 \\
\hline \multicolumn{6}{|l|}{ Ocupación (e) } \\
\hline Empleado contratado & 0,856 & 0,826 & 0,913 & & \\
\hline Desempleado & 0,866 & 1,026 & 0,889 & & \\
\hline Quehaceres del hogar & 0,805 & 1,117 & 0,834 & & \\
\hline Jubilado & 0,593 & 0,637 & $0,569(+)$ & & \\
\hline Estudiante & $0,251^{* *}$ & ** $0,232 * * *$ & $0,268 * * *$ & & \\
\hline \multicolumn{6}{|l|}{ Religión (f) } \\
\hline Católica & $2,489^{* *}=$ & $k * \quad 2,377 * * *$ & $2,634 * * *$ & $2,537 * * *$ & $2,738 * * *$ \\
\hline Evangélica & 1,687 & $1,863(+)$ & 1,616 & $1,847(+)$ & $1,806(+)$ \\
\hline Otras & 1,041 & 1,071 & 0,982 & 1,250 & 1,189 \\
\hline Constante & $0,032^{* *}$ & ** $0,121 * * *$ & $0,084 * * *$ & $0,021 * * *$ & $0,054 * * *$ \\
\hline Chi cuadrado & $120,720 * *=$ & $k * 69,441 * * *$ & $* 104,299 * * *$ & $107,732 * * *$ & * $91,900 * * *$ \\
\hline$-2 \log$ de verosimilitud final 1 . & $1.313,456 \quad 1$ & $1.366,344$ & $1.333,642$ & $1.334,592$ & $1.354,191$ \\
\hline R cuadrado de Cox y Snell & 0,095 & 0,056 & 0,083 & 0,085 & 0,073 \\
\hline R cuadrado de Nagelkerke & 0,137 & 0,080 & 0,119 & 0,122 & 0,105 \\
\hline Porcentaje pronosticado correcto & to 73,1 & 71,9 & 71,2 & 72,6 & 71,5 \\
\hline
\end{tabular}
$\mathrm{p} \leq 0,1$.

*** Significante al $\mathrm{p} \leq 0,01 . * *$ Significante al $\mathrm{p} \leq 0,05$. (+) Significante al

Nota: Las categorías de referencia son: (a) “mujer”; (b) “65 y más”; (c) "E”; (d) "estudios superiores”; (e) "trabajador independiente”; (f) "ninguna”.

Fuente: Centro de Estudios Públicos: "Estudio Social y de Opinión Pública No 11. Segunda Serie, Diciembre 1990” (Computer File: CEP0016-v1). 
TABLA N ${ }^{\circ}$ 3: $\quad$ MODELO DE REGRESIÓN LOGÍSTICA PARA 2005. “ODDS RATIO” DE LOS DETERMINANTES DE ADHESIÓN AL PDC (1= PDC, $0=$ OTROS)

\begin{tabular}{|c|c|c|c|c|c|}
\hline & Modelo 1 & Modelo 2 & Modelo 3 & Modelo 4 & Modelo 5 \\
\hline Sexo (a) & 1,181 & 1,245 & 1,179 & 1,043 & 1,050 \\
\hline \multicolumn{6}{|l|}{ Edad (b) } \\
\hline $18-24$ & 0,740 & 0,668 & 0,615 & 0,757 & 0,668 \\
\hline $25-34$ & 0,596 & $0,529(+)$ & $0,503(+)$ & 0,642 & $0,553(+)$ \\
\hline $35-44$ & 0,969 & 0,896 & 0,868 & 1,049 & 0,949 \\
\hline $45-54$ & 0,681 & 0,647 & 0,629 & 0,745 & 0,694 \\
\hline $55-64$ & 0,755 & 0,722 & 0,723 & 0,823 & 0,794 \\
\hline \multicolumn{6}{|l|}{ NSE (c) } \\
\hline ABC1 & 1,286 & 0,710 & & 1,217 & \\
\hline C2 & $2,984 * *$ & 1,793 & & $2,798 * *$ & \\
\hline C3 & 1,466 & 1,131 & & 1,394 & \\
\hline $\mathrm{D}$ & 1,164 & 1,088 & & 1,123 & \\
\hline \multicolumn{6}{|l|}{ Educación (d) } \\
\hline Sin estudios & 0,806 & & 0,501 & 0,867 & 0,538 \\
\hline Básica incompleta & $1,838(+)$ & & 1,245 & $1,938 * *$ & $1,309 * *$ \\
\hline Básica completa & $2,625 * * *$ & & $1,828 * *$ & $2,800 * * *$ & $1,932 * *$ \\
\hline Media incompleta & $2,327 * * *$ & & $1,680^{* *}$ & $2,419 * * *$ & 1,739 \\
\hline Media completa & 1,537 & & 1,240 & $1,584+$ & 1,262 \\
\hline \multicolumn{6}{|l|}{ Ocupación (e) } \\
\hline Empleado a tiempo completo & 0,776 & 0,722 & 0,839 & & \\
\hline Desempleado & 0,700 & 0,682 & 0,713 & & \\
\hline Quehaceres del hogar & 1,032 & 1,034 & 1,094 & & \\
\hline Jubilado & 0,688 & 0,619 & 0,723 & & \\
\hline Estudiante & 0,716 & 0,606 & 0,873 & & \\
\hline \multicolumn{6}{|l|}{ Religión (f) } \\
\hline Católica & 0,981 & 1,062 & 1,007 & 0,987 & 1,011 \\
\hline Evangélica & 0,642 & 0,731 & 0,621 & 0,634 & 0,612 \\
\hline Otras & $0,233 * *$ & $0,244 * * *$ & $* \quad 0,243 * *$ & $0,235 * *$ & $0,244^{* * *}$ \\
\hline Constante & $0,096 * * *$ & $0,192^{* * *}$ & $* * 0,175 * * *$ & $0,08 * * *$ & $0,148 * * *$ \\
\hline Chi cuadrado & $42,485 * * *$ & $* 30,015^{* *}$ & $33,213 * *$ & $39,919 * * *$ & * $30,995 * * *$ \\
\hline-2 log de verosimilitud final 1 . & $1.036,122$ & $1.055,378$ & $1.045,394$ & 1.038,688 & $1.047,612$ \\
\hline R cuadrado de Cox y Snell & 0,028 & 0,020 & 0,022 & 0,026 & 0,021 \\
\hline R cuadrado de Nagelkerke & 0,055 & 0,038 & 0,043 & 0,051 & 0,040 \\
\hline Porcentaje pronosticado correcto & 088,3 & 88,3 & 88,3 & 88,3 & 88,3 \\
\hline
\end{tabular}

*** Significante al $\mathrm{p} \leq 0,01$. ** Significante al $\mathrm{p} \leq 0,05$. (+) Significante al $\mathrm{p} \leq 0,1$.

Nota: Las categorías de referencia son: (a) "mujer"; (b) "65 y más"; (c) "E”; (d) "estudios superiores”; (e) "trabajador independiente”; (f) "ninguna”.

Fuente: Centro de Estudios Públicos: "Estudio Nacional de Opinión Pública No 23. Tercera Serie, Octubre - Noviembre 2005” (Computer File: CEP0051-v1). 
Hemos seleccionado sólo las encuestas de diciembre de 1990 y noviembre de 2005, a fin de comparar las tendencias desde el inicio de la "nueva democracia" hasta la última elección presidencial. Usamos la de diciembre de 1990 y no las anteriores debido a que es la encuesta que en ese año más se asimila en términos de categorías de respuestas a la de noviembre de $2005^{9}$, cuestión que facilita la comparación. Acá la crítica podría consistir en el sesgo de selección de las observaciones. Ante esto no podemos más que apelar a cuestiones de alcance de este trabajo. El objetivo consiste en establecer algunos determinantes relevantes a la hora de identificarse con el PDC, señalando las diferencias entre el inicio de la “nueva democracia” (1989) y la última elección de 2005.

Otro antecedente relevante, del que nos hacemos cargo, es que la medición de 1990 sólo considera las áreas urbanas, mientras que la de 2005 es una muestra nacional. Esto podría dificultar la comparación, pero no contamos con muestras nacionales en la serie completa. Para controlar este posible efecto, también realizamos modelos estadísticos con las variables mencionadas pero que, por razones de espacio y fluidez argumentativa, no mostramos. De todas formas, en tales modelos se mantienen los determinantes de adhesión que en 1990 tenía el PDC, aunque con algunas fluctuaciones en los coeficientes. Al considerar otras encuestas de la década, como por ejemplo la pre-electoral de 1999, la variable "religión" mantiene un peso significativo en la adhesión al PDC, tendencia válida para las mediciones anteriores, donde también resulta relevante el nivel educativo de los encuestados.

Una precisión previa a la presentación de los resultados. No incluimos la pregunta directa sobre intención de voto debido a que en 1990 no se contempló esta pregunta. Por tanto, a fin de tener las mismas variables dependientes para ambos modelos se optó por la pregunta sobre identificación y simpatía con el partido: “Ahora, de los siguientes partidos políticos que se presentan en esta tarjeta, ¿con cuál de ellos se identifica más o simpatiza más usted?”. Sin embargo, al construir los modelos con la pregunta sobre intención de voto para 2005 no se producen grandes variaciones. Además, por cuestiones relativas al sistema binominal, en algunos distritos los simpatizantes DC no tenían la posibilidad de votar por un candidato de sus filas, por lo que la pregunta sobre adhesión puede captar de mejor forma la cercanía con el partido.

\footnotetext{
${ }^{9}$ Por ejemplo, en las dos primeras mediciones de 1990 la categoría “jubilado” no figura dentro de las alternativas, categoría que sí aparece en la encuesta de diciembre de ese año.
} 
Para 1990 hay más claridad que para 2005 sobre los determinantes de adhesión al PDC. Existe mayor disposición para adherir al PDC en las personas con menor escolaridad, particularmente "básica incompleta", "básica completa” y "media incompleta", usando como categoría de referencia a aquellos con "estudios superiores". En el modelo 1, la probabilidad relativa de que una persona con estudios básicos incompletos adhiera al PDC es 4 veces más que si fuera otra con estudios superiores, manteniendo el resto de las variables constantes. Esta tendencia se mantiene en los cuatro modelos, variando los coeficientes entre 4,26 y 3,32.

Luego, por religión, observamos la mayor disposición de los “católicos” para adherir al PDC. Al igual que sucede con escolaridad, los coeficientes para esta categoría son significativos en todos los modelos. Por ejemplo, en el modelo 5, la probabilidad relativa de que un "católico" adhiera al PDC es 2,7 veces más que si no tuviera religión, manteniendo constante el resto de las variables. Los "evangélicos” también tienden a adherir más al PDC, aunque con coeficientes escasamente significativos. Esto nos retrata la prevalencia del factor religioso en la adhesión al partido, cuestión que no pudimos ver con claridad en los modelos lineales, particularmente en el de 1989.

Otros resultados que arroja el modelo es la tendencia de los estudiantes a no adherir al PDC. En los tres modelos donde figura la variable ocupación, son los estudiantes los que menos disposición tienen para adherir al partido, manteniendo el resto de las variables constante. Por edad, en tanto, aunque con menor claridad y significancia estadística, los que más adhieren al PDC son los encuestados entre " 25 y 35 años", aunque este dato no es concluyente debido a que la categoría mantiene alta significancia estadística sólo en dos de los cinco modelos.

Lo anterior cambia para la medición de 2005. En esta encuesta la adhesión al PDC, por educación, no marca el mismo nivel de diferencias entre las categorías de análisis y las de referencia. Así, la única que tiene significancia estadística en todos los modelos donde se ingresó la variable educación es "básica completa". De todas formas, y a pesar de estos cambios, la tendencia es similar a la de 1990: existe mayor disposición para adherir al PDC en las personas con menor nivel de escolaridad en comparación con los de "estudios superiores". Por ejemplo, para el modelo 4, la probabilidad relativa de que un encuestado con educación "básica completa" adhiera al PDC es 2,8 veces más que si tuviera "estudios superiores". 
Por religión se advierten los cambios más relevantes. A pesar de que en el modelo lineal por comunas el incremento del porcentaje de católicos provocaba un alza en la votación por el PDC (aunque discutimos este punto al ponderar los datos), en los modelos logísticos no existe evidencia respecto a que los católicos estén adhiriendo más al PDC en comparación con aquellos que no profesan “ninguna” religión”. De hecho, en ninguno de los modelos aparecen diferencias estadísticamente significativas. Lo contrario sucede con los que profesan "otras religiones", que tienen menor disposición para adherir al PDC cuando se mantiene constante el resto de las variables.

Si en la encuesta de 1990 el porcentaje de católicos que adherían al PDC era de 31\%, en 2005 esa cifra cae a 13,2\%. Esto, indudablemente, va ligado a la baja electoral del partido, aunque también es preciso señalar que su porcentaje de adhesión sólo se ubica 0,2 puntos más abajo que RN, que es el partido que más apoyo recibe de este grupo, apareciendo más atrás la UDI con $11,2 \%$. Por tanto, el argumento debe ser matizado. Si bien a nivel de comunas a mayor porcentaje de católicos, mayor porcentaje de votos para el PDC, en las encuestas esta relación baja, cuestión que también se debe a la exigua identificación partidaria que exhiben los chilenos en comparación con 1990, lo que no permite captar diferencias relevantes y que puede plantearse como un síntoma no sólo de desafección, sino que también de pérdida paulatina de sus antiguas bases de apoyo. Tal contexto explicaría, en parte, la caída en el respaldo de los católicos al PDC. Eso en términos de identificación. Otra interpretación nos sugiere que en 2005 se estrechan notoriamente las distancias entre la adhesión de "católicos” y quienes optan por "ninguna" religión, lo que en el modelo implica una pérdida de la significancia estadística de tales diferencias. De hecho, el porcentaje de "ninguna” religión que apoya al PDC es de 13,6 en 1990 y 11,3\% en 2005, mientras que el de católicos es, como dijimos, de 31\% para 1990 y 13,2\% para 2005. Una tercera interpretación, que considera los resultados de la última encuesta del Instituto de Ciencias Sociales de la Universidad Diego Portales (ICSO-UDP), muestra que el determinante religioso sigue siendo robusto respecto a la adhesión al PDC. Como esta encuesta es aplicada en sectores urbanos, bien podría ser que el hecho de ser católico impacte en la adhesión al PDC, particularmente en los últimos años, sólo en este tipo de localidades, mas no en las rurales debido, muy probablemente, al incremento de otras religiones como los evangélicos. Esto, además, sería concordante con los resultados de la encuesta CEP de 1990 que sólo consideró una muestra urbana. 


\section{La votación del PDC y su impacto en el desempeño electoral de Ricardo Lagos y Michelle Bachelet}

La segunda cuestión que abordamos, luego del análisis de los determinantes de adhesión, es en qué medida la votación de los dos candidatos presidenciales recientes de la Concertación (Lagos y Bachelet) es explicada por la votación DC. ¿Es verdad, acaso, que la DC aporta menos que el PS y el PPD cuando los candidatos presidenciales del bloque no son militantes del partido? En las dos primeras elecciones de la "Nueva Democracia” el coeficiente de correlación entre la votación del partido y la de Patricio Aylwin fue de 0,456 (***), mientras que con Eduardo Frei fue de 0,265 $(* * *)$. Esto evidenció el incremento de la votación de los candidatos presidenciales en la medida en que también lo hacía el voto por el PDC, cuestión que ya había sido contrastada para el caso de Aylwin en el trabajo de Scully y Valenzuela (1993). Como veremos, cuando el candidato presidencial de la Concertación no es militante del partido, tales coeficientes disminuyen. Esto se clarifica de mejor forma en los comicios concurrentes de 2005, instancia en que Bachelet obtuvo 5,8 puntos menos que la lista de diputados de la Concertación. Tal diferencia es suficientemente relevante como para hipotetizar sobre el "voto cruzado" que, presumiblemente, practicaron en mayor medida los votantes del PDC. Si bien el trabajo no profundiza en el "voto cruzado”, sí lo dejamos como antecedente válido para la interpretación de los datos ${ }^{10}$.

Antes de comenzar con el análisis mostramos la evolución de la distribución ideológica de los simpatizantes DC en el eje derecha-centroizquierda a partir de los resultados de las encuestas del CEP (ver Figura $\mathrm{N}^{\mathrm{0}}$ 4). Acá se evidencia el incremento paulatino de las posiciones de izquierda desde 2002 en adelante aunque con ciertas fluctuaciones ${ }^{11}$. Sin embargo, el período en que los simpatizantes DC más adhirieron a la izquierda fue en 1993, cuando finalizaba el mandato de Patricio Aylwin, lo que se explica por el ambiente de la post-transición y la identificación de la opción “derecha” con la figura del general Pinochet. En el gobierno de Eduardo Frei RuizTagle, en tanto, la opción “derecha” alcanzó su máximo dentro de la serie, mientras que bajo la administración Lagos esta alternativa y la "izquierda" se mantuvieron entre $30 \%$ y $40 \%$. De todas formas, aunque con variaciones,

${ }^{10}$ Existe una amplia literatura sobre el voto cruzado. Entre otros, ver Campbell y Miller, 1957; Cain et al., 1987; Jacobson, 1990; Fiorina, 1992; Nohlen, 1995; Taagepera y Grofman, 2003.

${ }^{11}$ Se suman las alternativas derecha y centro derecha, al igual que izquierda y centro izquierda. 

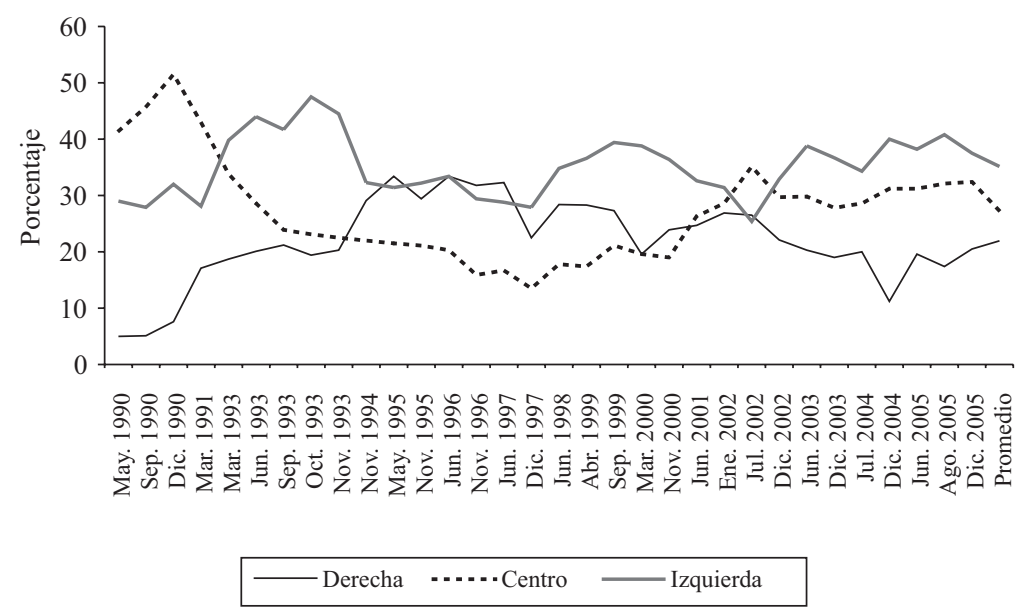

Fuente: encuestas CEP.

el electorado DC parece revalorar la opción del "centro", particularmente desde mediados de 2002. El promedio que alcanza esta alternativa es del 27\%, mientras que las opciones de izquierda lograron poco más del $35 \%$. Este argumento cobra mayor sentido al analizar los resultados según la escala política de 1 (“muy de izquierda”) a 10 (“muy de derecha”). Acá el encuestado tiene la posibilidad de definir más claramente sus opciones. En este caso, hemos considerado el estudio que realiza el ICSO-UDP para 2006. Según tales resultados, los adherentes al PDC se "auto-ubican" preferentemente al centro considerando la sumatoria de las opciones " 5 " y " 6 ", que totalizan el 54,2\%. La alternativa con mayores menciones es " 5 ”, que es la definición más comúnmente asociada a posturas de centro. Acá se autoubica el 36,6\% de los adherentes al PDC. Así, a pesar de la configuración bipolar del sistema de partidos chileno (Tironi y Agüero, 1999), los simpatizantes DC parecen buscar opciones de centro, cuestión que también se da en el total de electores. De esta forma, cobra mayor solidez la postura relativa a la vigencia de los antiguos tercios (Scully y Valenzuela, 1993), pero sin desconocer la convivencia con el sistema coalicional, también conocido como los “medios” (Garrido y Navia, 2005).

Luego de este panorama general, en las Tablas $\mathrm{N}^{\circ} 4$ y 5 mostramos los modelos de regresión múltiple de la votación de Lagos y Lavín en 19992000 y el porcentaje de votación de los partidos de la Concertación en las 
TABLA N ${ }^{\circ}$ 4: REGRESIÓN LINEAL DE VOTACIÓN DE LOS PARTIDOS POLÍTICOS DE LA CONCERTACIÓN EN 1996 SOBRE LA VOTACIÓN DE RICARDO LAGOS Y JOAQUÍN LAVÍN EN 1999-2000 ( $1^{\text {a }}$ Y 2 ${ }^{\mathrm{a}}$ VUELTA)

\begin{tabular}{|c|c|c|c|c|}
\hline & $\begin{array}{l}\text { Votación Lagos } \\
\text { primera vuelta }\end{array}$ & $\begin{array}{l}\text { Votación Lagos } \\
\text { segunda vuelta }\end{array}$ & $\begin{array}{l}\text { Votación Lavín } \\
\text { primera vuelta }\end{array}$ & $\begin{array}{l}\text { Votación Lavín } \\
\text { segunda vuelta }\end{array}$ \\
\hline Votación PDC 1996 & $\begin{array}{l}0,137 * * * \\
0,228 * * * \\
3,268 \\
(0,042)\end{array}$ & $\begin{array}{l}0,161 * * * \\
0,264 * * * \\
3,723 \\
(0,043)\end{array}$ & $\begin{array}{l}-0,150 * * * \\
-0,260 * * * \\
-3,304 \\
(0,045)\end{array}$ & $\begin{array}{l}-0,161 * * * \\
-0,264 * * * \\
-3,723 \\
(0,043)\end{array}$ \\
\hline Votación PS 1996 & $\begin{array}{l}0,298 * * * \\
0,316^{* * *} \\
6,398 \\
(0,047)\end{array}$ & $\begin{array}{l}0,322^{* * *} \\
0,350 * * * \\
6,713 \\
(0,048)\end{array}$ & $\begin{array}{l}-0,327 * * * \\
-0,348 * * * \\
-6,491 \\
(0,05)\end{array}$ & $\begin{array}{c}-0,322 * * * \\
-0,350 * * * \\
-6,713 \\
(0,048)\end{array}$ \\
\hline Votación PPD 1996 & $\begin{array}{l}0,125 * * \\
0,250 * * * \\
2,499 \\
(0,050)\end{array}$ & $\begin{array}{c}0,163 * * * \\
0,300 * * * \\
3,166 \\
(0,051)\end{array}$ & $\begin{array}{l}-0,145^{* * *} \\
-0,292 * * * \\
-2,683 \\
(0,054)\end{array}$ & $\begin{array}{l}-0,163 * * * \\
-0,300 * * * \\
-3,166 \\
(0,051)\end{array}$ \\
\hline Votación PRSD 1996 & $\begin{array}{l}0,255^{* * *} \\
0,210^{* * *} \\
4,291 \\
(0,099)\end{array}$ & $\begin{array}{l}0,287 * * * \\
0,239 * * * \\
4,699 \\
(0,061)\end{array}$ & $\begin{array}{l}-0,284 * * * \\
-0,230 * * * \\
-4,424 \\
(0,064)\end{array}$ & $\begin{array}{l}-0,287 * * * \\
-0,239 * * * \\
-4,699 \\
(0,061)\end{array}$ \\
\hline Constante & $\begin{array}{l}37,301 * * * \\
34,211 * * * \\
20,422 \\
(1,826)\end{array}$ & $\begin{array}{l}38,784 * * * \\
35,509 * * * \\
20,632 \\
(1,880)\end{array}$ & $\begin{array}{l}59,749 * * * \\
63,039 * * * \\
30,277 \\
(1,973)\end{array}$ & $\begin{array}{l}61,216 * * * \\
64,491 * * * \\
32,565 \\
(1,880)\end{array}$ \\
\hline $\begin{array}{l}\mathrm{R}^{2} \\
\mathrm{R}^{2}\end{array}$ & 0,183 & 0,203 & 0,189 & $\begin{array}{l}0,203 \\
0,190\end{array}$ \\
\hline $\begin{array}{l}\mathrm{R}^{2} \text { corregido } \\
\mathrm{F}\end{array}$ & $\begin{array}{c}0,169 \\
13,496 * * *\end{array}$ & $\begin{array}{c}0,190 \\
15,371^{* * *}\end{array}$ & $\begin{array}{c}0,175 \\
14,009 * * *\end{array}$ & $\begin{array}{c}0,190 \\
15,371^{* * *}\end{array}$ \\
\hline Durbin Watson & 1,934 & 1,957 & 1,957 & 1,957 \\
\hline $\mathrm{N}$ & 246 & 246 & 246 & 246 \\
\hline
\end{tabular}

*** Significante al $\mathrm{p} \leq 0,01$. ** Significante al $\mathrm{p} \leq 0,05$.

Nota: En primer lugar figuran los coeficientes beta no estandarizados, luego el coeficiente beta no estandarizado con datos ponderados, en tercer lugar el valor de la prueba t y finalmente, entre paréntesis, el error típico del coeficiente beta no ponderado. El resultado de tolerancia para el PDC es de 0,807, para el PS de 0,857, para el PPD de 0,828 y para el PRSD de 0,934.

Fuente: Elaboración propia con datos de www.elecciones.gov.cl 
TABLA N ${ }^{\circ}$ 5: $\quad$ REGRESIÓN LINEAL DE LA VOTACIÓN DE LOS PARTIDOS POLÍTICOS DE LA CONCERTACIÓN 1997 SOBRE LA VOTACIÓN DE RICARDO LAGOS Y JOAQUÍN LAVÍN EN 1999-2000 ( $1^{\text {a }}$ Y $2^{\text {a }}$ VUELTA)

\begin{tabular}{|c|c|c|c|c|}
\hline & $\begin{array}{l}\text { Votación Lagos } \\
\text { primera vuelta }\end{array}$ & $\begin{array}{l}\text { Votación Lagos } \\
\text { segunda vuelta }\end{array}$ & $\begin{array}{l}\text { Votación Lavín } \\
\text { primera vuelta }\end{array}$ & $\begin{array}{l}\text { Votación Lavín } \\
\text { segunda vuelta }\end{array}$ \\
\hline Votación PDC 1997 & $\begin{array}{l}0,382 * * * \\
0,285 * * * \\
6,884 \\
(0,056)\end{array}$ & $\begin{array}{l}0,419 * * * \\
0,319 * * * \\
7,191 \\
(0,058)\end{array}$ & $\begin{array}{l}-0,408 * * * \\
-0,313 * * * \\
-6,739 \\
(0,061)\end{array}$ & $\begin{array}{l}-0,419 * * * \\
-0,319 * * * \\
-7,191 \\
(0,058)\end{array}$ \\
\hline $\begin{array}{l}\text { Sub-bloque PS-PPD- } \\
\text { PRSD } 1997\end{array}$ & $\begin{array}{l}0,388^{* * *} \\
0,314 * * * \\
9,817 \\
(0,04)\end{array}$ & $\begin{array}{l}0,426 * * * \\
0,350 * * * \\
10,325 \\
(0,041)\end{array}$ & $\begin{array}{l}-0,417 * * * \\
-0,349 * * * \\
-9,724 \\
(0,043)\end{array}$ & $\begin{array}{c}-0,426 * * * \\
-0,350 * * * \\
-10,325 \\
(0,041)\end{array}$ \\
\hline Constante & $\begin{array}{l}27,247 * * * \\
32,770 * * * \\
12,433 \\
(2,191)\end{array}$ & $\begin{array}{l}28,180 * * * \\
34,313 * * * \\
12,332 \\
(2,285)\end{array}$ & $\begin{array}{l}70,207 * * * \\
64,386 * * * \\
29,551 \\
(2,376)\end{array}$ & $\begin{array}{l}71,820 * * * \\
65,687 * * * \\
31,429 \\
(2,285)\end{array}$ \\
\hline $\begin{array}{l}\mathrm{R}^{2} \\
\mathrm{R}^{2} \text { corregido } \\
\mathrm{F} \\
\text { Durbin Watson } \\
\mathrm{N}\end{array}$ & $\begin{array}{c}0,252 \\
0,247 \\
49,336 * * * \\
1,937 \\
296\end{array}$ & $\begin{array}{c}0,271 \\
0,266 \\
54,561 \text { *** } \\
1,930 \\
296\end{array}$ & $\begin{array}{c}0,248 \\
0,243 \\
48,334 * * * \\
1,926 \\
296\end{array}$ & $\begin{array}{c}0,271 \\
0,266 \\
54,561 * * * \\
1,930 \\
296\end{array}$ \\
\hline
\end{tabular}

*** Significante al $\mathrm{p} \leq 0,01$.

Nota: En primer lugar figuran los coeficientes beta no estandarizados, luego el coeficiente beta no estandarizado con datos ponderados, en tercer lugar el valor de la prueba $\mathrm{t}$ y finalmente, entre paréntesis, el error típico del coeficiente beta no ponderado. El resultado de tolerancia para el PDC y el sub-bloque PS-PP-PRSD es de 0,675.

Fuente: Elaboración propia con datos de www.elecciones.gov.cl

municipales de 1996 y las parlamentarias de 1997. Los coeficientes de ambos modelos indican un impacto positivo de la votación PDC sobre Lagos, aunque inferiores al resto de los partidos de la Concertación. Para el análisis por votación en las parlamentarias, consideramos al PDC y al bloque progresista integrado por el PS-PPD y PRSD. Conformamos este sub-bloque debido a que en las elecciones parlamentarias, a raíz de las omisiones propias de un sub-pacto, existe una alta cantidad de casos perdidos, lo que no permite un correcto análisis de regresión lineal múltiple.

De las Tablas $\mathrm{N}^{\circ} 4$ y $\mathrm{N}^{\circ} 5$ se desprende que el efecto de la votación por los partidos de la Concertación sobre el porcentaje de Lagos es mayor 
en las parlamentarias 1997 que en las municipales 1996. Aunque los valores del efecto de la votación partidista tienen signos similares en ambos modelos, los coeficientes de las elecciones parlamentarias parecen tener una mejor capacidad explicativa. No obstante, a partir de los resultados de la contienda municipal de 1996 y parlamentaria de 1997, el efecto y relación que tiene la votación por el PDC en la votación de Lagos tanto en primera como en segunda vuelta tiene un coeficiente inferior al observado para el resto de los partidos de la Concertación. Otro punto a destacar es que el PDC es el partido de la Concertación que presenta el coeficiente negativo más débil con respecto a Lavín tanto en la primera como en la segunda vuelta. Más allá de estas consideraciones, donde concluimos que el PDC es el partido que menos aporta a la votación de Lagos dentro de la Concertación, debemos mantener cierta cautela con el modelo de regresión. Esto porque para las parlamentarias de 1997 sumamos las votaciones del PS, PPD y PRSD en el denominado sub-bloque progresista. A fin de clarificar tal situación mostramos las correlaciones (Tabla $\mathrm{N}^{\mathrm{o}}$ 6) entre la votación de cada partido de la Concertación con los apoyos a Lagos:

TABLA No 6:

CORRELACIÓN ENTRE LA VOTACIÓN DE LOS PARTIDOS POLÍTICOS DE LA CONCERTACIÓN Y DE LA ALIANZA EN 1997 CON LA VOTACIÓN DE RICARDO LAGOS Y JOAQUÍN LAVÍN EN 1999-2000 (1ª Y 2a VUELTA)

\begin{tabular}{|c|c|c|c|c|}
\hline & $\begin{array}{l}\text { Votación Lagos } \\
\text { primera vuelta }\end{array}$ & $\begin{array}{l}\text { Votación Lagos } \\
\text { segunda vuelta }\end{array}$ & $\begin{array}{l}\text { Votación Lavín } \\
\text { primera vuelta }\end{array}$ & $\begin{array}{l}\text { Votación Lavín } \\
\text { segunda vuelta }\end{array}$ \\
\hline Votación PDC 1997 & $\begin{array}{l}0,079 \\
0,031 * * *\end{array}$ & $\begin{array}{l}0,081 \\
0,038 * * *\end{array}$ & $\begin{array}{l}-0,077 \\
0,034 * * *\end{array}$ & $\begin{array}{l}-0,081 \\
-0,038 * * *\end{array}$ \\
\hline & 300 & 300 & 297 & 300 \\
\hline Votación PS 1997 & $\begin{array}{r}0,456 * * * \\
0,422 * * * \\
146\end{array}$ & $\begin{array}{r}0,461 * * * \\
0,413 * * * \\
146\end{array}$ & $\begin{array}{l}-0,453 * * * \\
-0,416 * * * \\
143\end{array}$ & $\begin{array}{l}-0,461 * * * \\
-0,413^{* * *} \\
146\end{array}$ \\
\hline Votación PPD 1997 & $\begin{array}{r}0,200^{* * *} \\
0,306 * * * \\
169\end{array}$ & $\begin{array}{c}0,224 * * \\
0,322 * * * \\
169\end{array}$ & $\begin{array}{l}-0,203 * * * \\
-0,315 * * * \\
169\end{array}$ & $\begin{array}{l}-0,224 * * * \\
-0,322 * * * \\
169\end{array}$ \\
\hline Votación PRSD 1997 & $\begin{array}{l}0,284 * * \\
0,290 * * * \\
60\end{array}$ & $\begin{array}{l}0,242(+) \\
0,230 * * * \\
60\end{array}$ & $\begin{array}{l}-0,247(+) \\
-0,227 * * * \\
60\end{array}$ & $\begin{array}{l}-0,242(+) \\
-0,230 * * * \\
60\end{array}$ \\
\hline
\end{tabular}

Nota: En primer lugar figuran los coeficientes de correlación sin ponderar, luego los coeficientes de correlación ponderados según votos emitidos en las parlamentarias de 1997 y luego el número de comunas en las que compitió cada partido.

Fuente: Elaboración propia con datos de www.elecciones.gov.cl 
En la Tabla $\mathrm{N}^{\circ} 6$ observamos el bajísimo coeficiente de correlación entre la votación del PDC con la de Ricardo Lagos. Si bien el coeficiente es superior a cero, éste no es estadísticamente significativo. Cuando ponderamos los datos por cantidad de votos emitidos, el coeficiente, incluso, baja. Esto se debe al impacto que generan las comunas más grandes sobre el total. Es decir, en aquellas comunas grandes donde la DC era fuerte, Lagos no tenía un respaldo de la misma magnitud. Esto no necesariamente nos hace suponer que parte de la votación por el PDC se fuera a Lavín. Y acá una aclaración relevante. Probablemente, en aquellas comunas donde el PDC era fuerte, también la derecha obtenía altas votaciones, sucediendo lo contrario con el PPD y el PS. Es decir, la votación del PDC, al estar negativamente correlacionada con los partidos del bloque "progresista", puede generar una interpretación alternativa: no es que el PDC haya apoyado menos a Lagos que los otros partidos de la Concertación. Simplemente, en aquellas comunas donde la DC era fuerte, también lo era la derecha. Así, se evitaba la "súper votación" que obtenía Lagos en comunas donde había un bloque PS-PPD fuerte al que se sumaba el PDC. Por ende, en esas comunas donde había alta votación para el PDC y también para la derecha, la correlación con el voto por Lagos era débil, inexistente o, incluso, negativa.

De lo anterior se desprenden dos cuestiones relevantes: 1) como señalamos, el PDC es el partido que presenta la más baja relación entre su votación y la obtenida por Ricardo Lagos. En el modelo de regresión, de hecho, con los datos de las municipales los coeficientes siguen siendo bajos en comparación con el resto de los partidos concertacionistas exceptuando el PPD. 2) En el análisis de las parlamentarias 1997 y su impacto en la votación de Lagos existen, al menos, dos interpretaciones. Primero, que la votación del PDC pronostica en menor medida el apoyo obtenido por Lagos en comparación con el bloque progresista. Segundo, a partir del análisis de correlaciones, la votación del PDC no permite afirmar con un alto grado de certeza estadística que, efectivamente, un incremento en su votación implicó también un incremento en la de Lagos, existiendo importantes diferencias con el resto de los partidos de la Concertación.

Para complementar estos datos mostramos una tabla de contingencia (Tabla $\mathrm{N}^{\circ}$ 7) con la preferencia por candidato presidencial e identificación partidaria. Esto nos permite generar un análisis con una unidad más específica: las personas. Esta tabla deja en evidencia el menor respaldo de los simpatizantes del PDC hacia Ricardo Lagos en comparación con los del PS y del PPD ${ }^{12}$. Esto es concordante con los resultados anteriores. Si bien las

${ }^{12}$ Hemos dejado fuera al PRSD debido al escaso número de menciones que recibe en la encuesta. 
TABLA N ${ }^{\circ}$ 7: $\quad$ EL APOYO A LOS CANDIDATOS PRESIDENCIALES DE 1999 SEGÚN PARTIDO POLÍTICO ${ }^{1}$

\begin{tabular}{|c|c|c|c|c|c|c|c|c|}
\hline & Lagos & Lavín & $\begin{array}{l}\text { Vota en } \\
\text { blanco }\end{array}$ & $\begin{array}{l}\text { Anula } \\
\text { el voto }\end{array}$ & $\begin{array}{c}\text { No } \\
\text { votará/ } \\
\text { no está } \\
\text { inscrito } \\
\text { para votar }\end{array}$ & $\begin{array}{c}\text { No sabe, } \\
\text { no está } \\
\text { decidido }\end{array}$ & $\begin{array}{c}\text { No } \\
\text { contesta }\end{array}$ & Total \\
\hline \multirow[t]{2}{*}{ PDC } & 152 & 46 & 4 & 6 & 6 & 14 & 6 & 238 \\
\hline & $63,9 \%$ & $19,3 \%$ & $1,7 \%$ & $2,5 \%$ & $2,5 \%$ & $5,9 \%$ & $2,5 \%$ & $100 \%$ \\
\hline \multirow[t]{2}{*}{ PS } & 99 & 4 & 2 & 0 & 0 & 1 & 0 & 114 \\
\hline & $86,8 \%$ & $3,5 \%$ & $1,8 \%$ & $0 \%$ & $0 \%$ & $0,9 \%$ & $0 \%$ & $100 \%$ \\
\hline \multirow[t]{2}{*}{ PPD } & 119 & 20 & 2 & 2 & 10 & 3 & 3 & 160 \\
\hline & $74,4 \%$ & $12,5 \%$ & $1,3 \%$ & $1,3 \%$ & $6,3 \%$ & $1,9 \%$ & $1,9 \%$ & $100 \%$ \\
\hline
\end{tabular}

${ }^{1}$ La pregunta sobre apoyo a partidos es: “Ahora, de los siguientes partidos políticos que se presentan en esta tarjeta, ¿con cuál de ellos se identifica más o simpatiza más usted?” La pregunta por intención de voto, en tanto, es la siguiente: “Si las elecciones para Presidente de la República fueran este domingo, y los candidatos que se presentan son los que aparecen en la lista, ¿Por cuál vota usted?”.

Fuente: Centro de Estudios Públicos: "Estudio Nacional de Opinión Pública No 10, Septiembre - Octubre 1999 (Computer File: CEP0038-v1).

unidades de análisis cambian, las interpretaciones son complementarias. En este caso, vemos que los adherentes al PDC tuvieron menos disposición a votar por Lagos al compararlos con los del PS y el PPD.

Luego de haber revisado las características del voto por el PDC y su impacto en la votación de Lagos y Lavín para 1999, resta analizar el rol que jugó este partido en la elección presidencial de 2005.

A pesar de haber quedado sin candidatura a la Presidencia, el PDC logró una negociación favorable. Formalmente, compitió en 56 distritos, dejando cuatro cupos para los independientes por el partido. El resultado no fue del todo satisfactorio, pues redujo su número de diputados y de senadores. Esto fue un tanto sorprendente dado el desempeño que mostró el partido en las municipales de 2004. Precisamente, en el siguiente modelo (Tabla $\mathrm{N}^{\circ} 8$ ) vemos el impacto que tuvo su votación sobre los candidatos presidenciales de la Alianza y de la Concertación en la primera vuelta de diciembre de 2005. 
TABLA N ${ }^{\circ}$ 8: $\quad$ REGRESIÓN LINEAL DE VOTACIÓN DE LOS PARTIDOS POLÍTICOS DE LA CONCERTACIÓN 2004 SOBRE LA VOTACIÓN DE MICHELLE BACHELET, JOAQUÍN LAVÍN Y SEBASTIÁN PIÑERA EN 2005-2006 (1ª Y 2a VUELTA)

\begin{tabular}{|c|c|c|c|c|c|}
\hline & $\begin{array}{c}\text { Votación } \\
\text { Bachelet } \\
\text { primera } \\
\text { vuelta }\end{array}$ & $\begin{array}{c}\text { Votación } \\
\text { Piñera } \\
\text { primera } \\
\text { vuelta }\end{array}$ & $\begin{array}{l}\text { Votación } \\
\text { Lavín } \\
\text { primera } \\
\text { vuelta }\end{array}$ & $\begin{array}{c}\text { Votación } \\
\text { Bachelet } \\
\text { segunda } \\
\text { vuelta }\end{array}$ & $\begin{array}{l}\text { Votación } \\
\text { Piñera } \\
\text { segunda } \\
\text { vuelta }\end{array}$ \\
\hline \multirow[t]{4}{*}{ Votación PDC 2004} & $0,168 * * *$ & $-0,083(+)$ & $-0,097(+)$ & $0,215^{* * *}$ & $-0,215 * * *$ \\
\hline & $0,288 * * *$ & $-0,067 * * *$ & $-0,222 * * *$ & $0,343^{* * *}$ & $-0,343 * * *$ \\
\hline & 2,588 & $-1,721$ & $-1,819$ & 3,108 & $-3,108$ \\
\hline & $(0,065)$ & $(0,048)$ & $(0,053)$ & $(0,069)$ & $(0,069)$ \\
\hline \multirow[t]{4}{*}{ Votación PS 2004} & $0,501 * * *$ & $-0,194 * * *$ & $-0,380 * * *$ & $0,555^{* * *}$ & $-0,555 * * *$ \\
\hline & $0,577 * * *$ & $-0,265 * * *$ & $-0,390 * * *$ & $0,665^{* * *}$ & $-0,665 * * *$ \\
\hline & 5,829 & $-3,038$ & $-5,375$ & 6,032 & $-6,032$ \\
\hline & $(0,086)$ & $(0,064)$ & $(0,071)$ & $(0,092)$ & $(0,092)$ \\
\hline \multirow[t]{4}{*}{ Votación PPD 2004} & $0,269 * * *$ & $-0,044$ & $-0,252 * * *$ & $0,319 * * *$ & $-0,319 * * *$ \\
\hline & $0,388 * * *$ & $-0,168 * * *$ & $-0,246 * * *$ & $0,449 * * *$ & $-0,449 * * *$ \\
\hline & 3,471 & $-0,759$ & $-3,962$ & 3,855 & $-3,855$ \\
\hline & $(0,077)$ & $(0,057)$ & $(0,064)$ & $(0,083)$ & $(0,083)$ \\
\hline \multirow[t]{4}{*}{ Votación PRSD 2004} & 0,098 & $-0,016$ & $-0,019$ & 0,098 & $-0,098$ \\
\hline & $0,325 * * *$ & $-0,139 * * *$ & $-0,054 * * *$ & $0,368 * * *$ & $-0,368 * * *$ \\
\hline & 0,824 & $-0,187$ & $-0,193$ & 0,769 & $-0,769$ \\
\hline & $(0,119)$ & $(0,088)$ & $(0,098)$ & $(0,127)$ & $(0,127)$ \\
\hline \multirow[t]{4}{*}{ Constante } & $35,257 * * *$ & $29,331 * * *$ & $31,863 * * *$ & $40,071^{* * *}$ & $59,929 * * *$ \\
\hline & $28,109 * * *$ & $32,222 * * *$ & $34,789 * * *$ & $32,559 * * *$ & $67,441 * * *$ \\
\hline & 15,359 & 17,216 & 16,891 & 16,312 & 24,396 \\
\hline & $(2,296)$ & $(1,704)$ & $(1,886)$ & $(2,457)$ & $(2,457)$ \\
\hline $\mathrm{R}^{2}$ & 0,145 & 0,043 & 0,138 & 0,161 & 0,161 \\
\hline $\mathrm{R}^{2}$ corregido & 0,130 & 0,027 & 0,124 & 0,147 & 0,147 \\
\hline $\mathrm{F}$ & $10,113^{* * *}$ & $2,676 * *$ & $9,586 * * *$ & $11,459 * * *$ & $11,459 * * *$ \\
\hline Durbin Watson & 1,927 & 1,859 & 1,960 & 1,960 & 1,960 \\
\hline $\mathrm{N}$ & 244 & 244 & 244 & 244 & 244 \\
\hline
\end{tabular}

*** Significante al $\mathrm{p} \leq 0,01$. ** Significante al $\mathrm{p} \leq 0,05$. (+) Significante al $\mathrm{p} \leq 0,1$.

Nota: En primer lugar figuran los coeficientes beta no estandarizados, luego el coeficiente beta no estandarizado con datos ponderados, en tercer lugar el valor de la prueba t y finalmente, entre paréntesis, el error típico del coeficiente beta no ponderado. El resultado de tolerancia para el PDC es de 0,918, para el PS de 0,922, para el PPD de 0,900 y para el PRSD de 0,949.

Fuente: Elaboración propia con datos de www.elecciones.gov.cl 
En la Tabla $N^{\circ} 8$ podemos apreciar que el PDC es el partido que tiene el coeficiente más bajo, luego del PRSD, respecto a la votación por Bachelet en primera vuelta, el que crece para la elección de enero. Además, en la primera vuelta los coeficientes con Piñera y Lavín son negativos y, en el caso de Piñera, considerando datos ponderados y no ponderados, son muy cercanos a cero. Sin embargo, en la segunda vuelta los coeficientes con Piñera son negativos y altamente significativos para datos ponderados y no ponderados. Esto, muy intuitivamente aún, nos indicaría un antecedente para evaluar el eventual "voto estratégico" y "voto de castigo" que ejercieron los votantes DC. Asumiendo los riesgos de la "falacia ecológica”, es probable que algunos votantes del partido hayan sufragado por Piñera en la primera vuelta y por Bachelet en la segunda, evitando así el paso al segundo turno de Lavín, el candidato con mayor índice de rechazo. Otra interpretación nos sugiere que dichos adherentes emitieron un "voto de castigo" considerando que era la segunda vez consecutiva que el partido no llevaba candidato presidencial. Ambas interpretaciones pueden ser testeadas de mejor forma en un análisis más profundo de las encuestas de opinión.

La elección de 2004, además, fue muy particular debido a las modificaciones al sistema electoral, pues se eligieron separadamente alcaldes y concejales. En el modelo anterior mostramos cómo los porcentajes de votación de los partidos en la elección de concejales impactaban en el porcentaje de votos de Bachelet en primera vuelta. Como ahora también contamos con los datos de las elecciones de alcaldes, presentamos una matriz de correlaciones (Tabla $\mathrm{N}^{\mathrm{0}}$ 9) entre la votación obtenida por los alcaldes y la obtenida por Bachelet.

Como vemos en la Tabla $\mathrm{N}^{\circ}$ 9, los coeficientes de correlación entre el PDC y la votación de Bachelet en primera y segunda vuelta son muy similares a los de los restantes partidos de la Concertación, salvo el PRSD. Con esto podemos suponer que la presencia de alcaldes DC en las distintas comunas del país, particularmente en las más grandes debido al efecto de la ponderación, favoreció el caudal de votos que recibió Bachelet. Incluso, el coeficiente de correlación con la votación de Piñera es negativo y significativo para ambas vueltas. Así, no tenemos evidencia que respalde la hipótesis de que Bachelet no ganó en primera vuelta debido al escaso apoyo recibido en aquellas comunas gobernadas por alcaldes del PDC.

Veamos ahora qué sucedió con el impacto de las votaciones de los partidos de la Concertación en las parlamentarias 2005 sobre los apoyos a Bachelet (ver Tabla $N^{\circ} 10$ ). Nuevamente agrupamos en sub-pacto al PS-PPD y PRSD.

En la Tabla $N^{0} 10$, y al igual que en el modelo anterior, debemos ser cautelosos en la interpretación de datos. Ya sabemos que resulta inapropia- 
TABLA N ${ }^{\circ}$ 9: $\quad$ CORRELACIONES ENTRE EL PORCENTAJE DE VOTACIÓN DE LOS PARTIDOS DE LA CONCERTACIÓN EN LA ELECCIÓN DE ALCALDES 2004 Y EL PORCENTAJE DE VOTOS OBTENIDO POR MICHELLE BACHELET EN PRIMERA Y SEGUNDA VUELTA

\begin{tabular}{lcc}
\hline Porcentaje de votación & $\begin{array}{c}\text { Morcentaje de votación } \\
\text { Michelle Bachelet } \\
\text { primera vuelta } \\
\text { sichelle Bachelet } \\
\text { segunda vuelta }\end{array}$ \\
\hline PDC & $0,208^{* * *}$ & $0,215^{* * *}$ \\
& $0,252^{* * *}$ & $0,269^{* * *}$ \\
PPD & 158 & 158 \\
& 0,160 & 0,131 \\
& $0,285^{* * *}$ & $0,292^{* * *}$ \\
PS & 55 & 55 \\
& 0,131 & 0,185 \\
& $0,227^{* * *}$ & $0,248^{* * *}$ \\
PRSD & 75 & 76 \\
& $-0,077$ & 0,066 \\
& 0,002 & $0,112^{* * *}$ \\
& 75 & 75 \\
\hline
\end{tabular}

*** Significante al $\mathrm{p} \leq 0,01$.

Nota: En primer lugar figuran los coeficientes de correlación sin ponderar, luego los coeficientes de correlación ponderados según votos emitidos en las municipales de 2004 y luego el número de comunas en las que compitió cada partido.

Fuente: Elaboración propia con datos de www.elecciones.gov.cl

do calcular el impacto de cada partido por separado en la elección presidencial asumiendo un único modelo. Esto por los pactos de omisión entre el PS, PPD y PRSD y el consecuente incremento de los casos perdidos. Sin perjuicio de tal limitante, vemos que el PDC parece tener un impacto un tanto más robusto que sus compañeros de coalición sobre la votación de Bachelet en la primera vuelta, mas no en la segunda, observando sólo datos no ponderados. Incluso es el partido que "más inversamente" explica la votación por Piñera en la primera ronda. Los coeficientes del PDC y del sub-pacto PSPPD-PRSD son similares, por lo que no podríamos discriminar claramente la supremacía de uno sobre otro, aunque con los datos ponderados el coeficiente del PDC es más bajo. Para clarificar esta cuestión mostramos una 
TABLA N ${ }^{\circ}$ 10: $\quad$ REGRESIÓN LINEAL DE LA VOTACIÓN DE LOS PARTIDOS POLÍTICOS DE LA CONCERTACIÓN 2005 SOBRE LA VOTACIÓN DE MICHELLE BACHELET Y SEBASTIÁN PIÑERA EN 2005-2006 (1 $1^{\text {a }}$ 2 2a VUELTA)

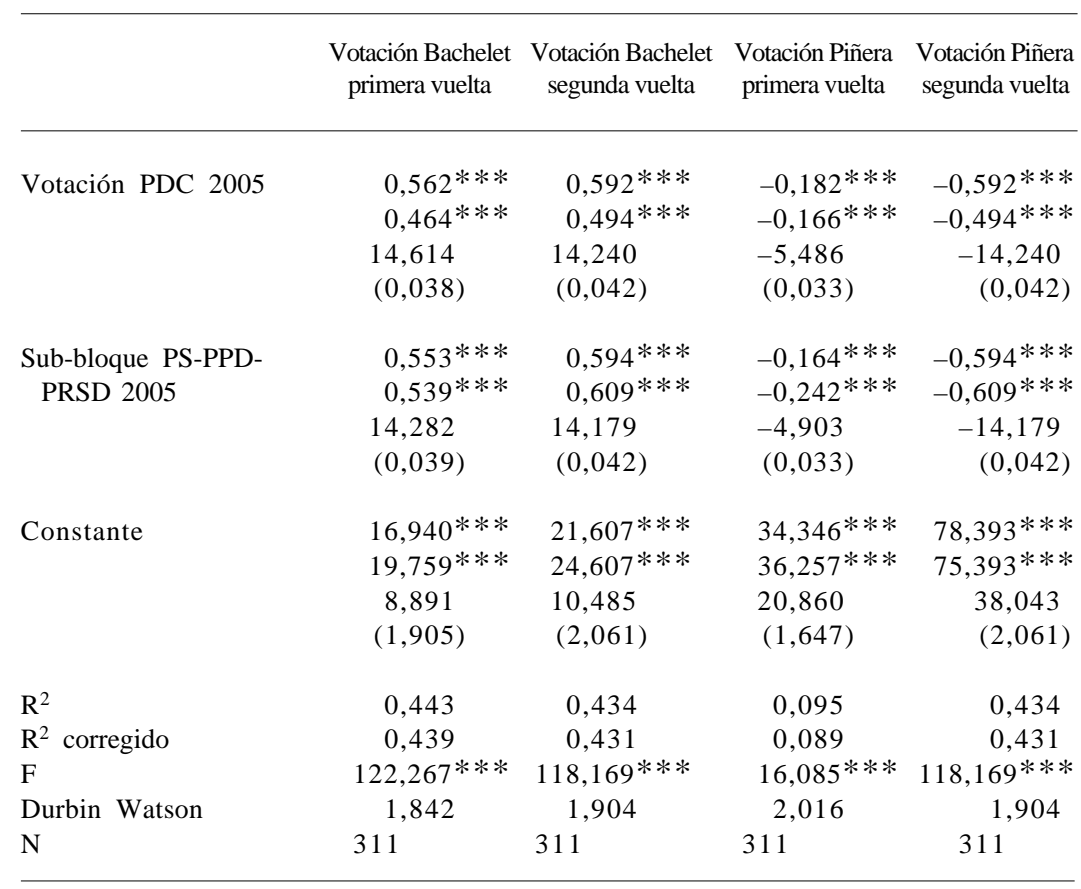

$* * *$ Significante al $\mathrm{p} \leq 0,01$.

Nota: En primer lugar figuran los coeficientes beta no estandarizados, luego el coeficiente beta no estandarizado con datos ponderados, en tercer lugar el valor de la prueba t y finalmente, entre paréntesis, el error típico del coeficiente beta no ponderado. El resultado de tolerancia para el PDC y el sub-bloque PS-PP-PRSD es de 0,498.

Fuente: Elaboración propia con datos de www.elecciones.gov.cl

matriz de correlaciones (Tabla $N^{0} 11$ ) con la votación de cada partido por separado.

En la Tabla N ${ }^{\circ} 11$ se advierten algunos aspectos relevantes. La votación DC está positivamente relacionada con la de Bachelet, pero cuando los datos se ponderan, el coeficiente cae significativamente, siendo muy inferior al del resto de los partidos de la Concertación, cuestión que ya se advertía en el modelo lineal múltiple. Incluso, en la segunda vuelta es cercano a cero. De este modo, el rendimiento del PDC en las comunas más grandes no fue de la misma magnitud que el de Bachelet. De aquí que encuentre asidero la hipótesis del "voto cruzado", principalmente emitido por votantes 
TABLA N ${ }^{\circ}$ 11: CORRELACIÓN ENTRE LA VOTACIÓN DE LOS PARTIDOS POLÍTICOS DE LA CONCERTACIÓN Y ALIANZA EN 2005 Y LA VOTACIÓN DE MICHELLE BACHELET Y SEBASTIÁN PIÑERA EN 2005-2006 ( $\left(1^{\text {a }}\right.$ Y $2^{a}$ VUELTA) Y JOAQUíN LAVÍN EN 2005 (1ª VUELTA)

\begin{tabular}{|c|c|c|c|c|c|}
\hline & $\begin{array}{c}\text { Votación } \\
\text { Bachelet } \\
\text { primera } \\
\text { vuelta }\end{array}$ & $\begin{array}{c}\text { Votación } \\
\text { Bachelet } \\
\text { segunda } \\
\text { vuelta }\end{array}$ & $\begin{array}{l}\text { Votación } \\
\text { Lavín } \\
\text { primera } \\
\text { vuelta }\end{array}$ & $\begin{array}{c}\text { Votación } \\
\text { Piñera } \\
\text { primera } \\
\text { vuelta }\end{array}$ & $\begin{array}{c}\text { Votación } \\
\text { Piñera } \\
\text { segunda } \\
\text { vuelta }\end{array}$ \\
\hline \multirow[t]{3}{*}{ Votación PDC 2005} & $0,290 * * *$ & $0,275 * * *$ & $-0,145 * * *$ & $-0,166 * * *$ & $-0,275 * * *$ \\
\hline & $0,109 * * *$ & $0,064 * * *$ & $-0,063 * * *$ & $0,025 * * *$ & $-0,064 * * *$ \\
\hline & 327 & 327 & 327 & 327 & 327 \\
\hline \multirow[t]{3}{*}{ Votación PS 2005} & 0,141 & 0,139 & $-0,197 * *$ & $-0,052$ & $-0,139$ \\
\hline & $0,278 * * *$ & $0,299 * * *$ & $-0,266 * * *$ & $-0,260 * * *$ & $-0,299 * * *$ \\
\hline & 119 & 119 & 119 & 119 & 119 \\
\hline \multirow[t]{3}{*}{ Votación PPD 2005} & $0,277 * * *$ & $0,306 * * *$ & $-0,256 * * *$ & $-0,131$ & $-0,306 * * *$ \\
\hline & $0,504 * * *$ & $0,514 * * *$ & $-0,470 * * *$ & $-0,383 * * *$ & $-0,514 * * *$ \\
\hline & 144 & 144 & 144 & 144 & 144 \\
\hline \multirow[t]{3}{*}{ Votación PRSD 2005} & $0,212(+)$ & 0,193 & $-0,202(+)$ & 0,019 & $-0,193$ \\
\hline & $0,205^{* * *}$ & $0,180 * * *$ & $0,019 * * *$ & $-0,195 * * *$ & $-0,180 * * *$ \\
\hline & 67 & 67 & 67 & 67 & 67 \\
\hline
\end{tabular}
$\mathrm{p} \leq 0,1$.

*** Significante al $\mathrm{p} \leq 0,01$. ** Significante al $\mathrm{p} \leq 0,05$. (+) Significante al

Nota: En primer lugar figuran los coeficientes de correlación sin ponderar, luego los coeficientes de correlación ponderados según votos emitidos en las parlamentarias de 2005 y luego el número de comunas en las que compitió cada partido.

Fuente: Elaboración propia con datos de www.elecciones.gov.cl

del partido. Es decir, electores que apoyaron candidatos a diputados del PDC, pero que no votaron por Bachelet en la presidencial.

Para respaldar esta interpretación mostramos una tabla de contingencia de la encuesta pre-electoral de 2005 sobre intención de voto por candidato y según simpatía por partido (Tabla Nº 12). Acá, al igual que con Lagos, también se observa la menor disposición de los DC a votar por Bachelet. Incluso, un 18,1\% de estos electores señaló su intención de votar por Piñera. De todas formas, el apoyo hacia Bachelet por parte de los simpatizantes DC fue mayor que el observado en la intención de voto por Lagos. En el PS y el PPD el porcentaje de apoyo hacia Bachelet fue superior, llegando a $81,4 \%$ y $73,9 \%$ respectivamente. 
TABLA N 12 :

EL APOYO A LOS CANDIDATOS PRESIDENCIALES DE 2005 SEGÚN PARTIDO POLÍTICO ${ }^{1}$

\begin{tabular}{|c|c|c|c|c|c|c|c|c|c|c|}
\hline & $\begin{array}{c}\text { Sebastián } \\
\text { Piñera }\end{array}$ & $\begin{array}{l}\text { Joaquín } \\
\text { Lavín }\end{array}$ & $\begin{array}{l}\text { Michelle } \\
\text { Bachelet }\end{array}$ & $\begin{array}{l}\text { Tomás } \\
\text { Hirsch }\end{array}$ & Blanco & Nulo & $\begin{array}{c}\text { No } \\
\text { votaría }\end{array}$ & $\begin{array}{l}\text { No } \\
\text { sabe }\end{array}$ & $\begin{array}{c}\text { No } \\
\text { contesta }\end{array}$ & Total \\
\hline \multirow[t]{2}{*}{ PDC } & 32 & 12 & 122 & 1 & 1 & 1 & 1 & 3 & 4 & 177 \\
\hline & $18,1 \%$ & $6,8 \%$ & $68,9 \%$ & $0,6 \%$ & $0,6 \%$ & $0,6 \%$ & $0,6 \%$ & $1,7 \%$ & $2,3 \%$ & $100 \%$ \\
\hline \multirow[t]{2}{*}{ PS } & 13 & 6 & 136 & 5 & 0 & 3 & 2 & 2 & 0 & 167 \\
\hline & $7,8 \%$ & $3,6 \%$ & $81,4 \%$ & $3 \%$ & $0 \%$ & $1,8 \%$ & $1,2 \%$ & $1,2 \%$ & $0 \%$ & $100 \%$ \\
\hline \multirow[t]{2}{*}{ PPD } & 29 & 9 & 139 & 3 & 3 & 0 & 0 & 4 & 1 & 188 \\
\hline & $15,4 \%$ & $4,8 \%$ & $73,9 \%$ & $1,6 \%$ & $1,6 \%$ & $0 \%$ & $0 \%$ & $2,1 \%$ & $0,5 \%$ & $100 \%$ \\
\hline
\end{tabular}

${ }^{1}$ Realizando el cruce con la pregunta específica sobre intención de voto por partido, el resultado por PDC es casi idéntico, siendo del 68,2\%, mientras que en el resto hay algunas variaciones. En el PS el apoyo a Bachelet alcanza en esta pregunta 87,5\% y en el PPD 78,6\%, marcando mayores diferencias con el PDC.

Fuente: Centro de Estudios Públicos: "Estudio Nacional de Opinión Pública № 23. Tercera Serie, Octubre - Noviembre 2005” (Computer File: CEP0051-v1).

La votación DC tuvo un impacto positivo leve en la votación lograda por Lagos y mayor en la de Bachelet, cuestión que también se refleja en los resultados de las encuestas pre-electorales. A pesar de las diferencias entre los coeficientes de correlación ponderados y no ponderados, el PDC parece aportar más a la candidatura de Bachelet que a la de Lagos. Además, existe una relación negativa entre las votaciones del PDC y Piñera en la segunda vuelta. La intensidad de los coeficientes nos podría conducir a otra interpretación asumiendo los riesgos de la falacia ecológica: probablemente, hubo votantes DC que sufragaron por Piñera en primera vuelta y que en el segundo turno apoyaron a Bachelet, y de ahí la ventaja que esta candidata lograra en dicha instancia. Además, en la misma encuesta pre-electoral del CEP, el apoyo de los adherentes DC a Bachelet en la segunda vuelta supera el $72 \%$ en un escenario simulado de competencia con Piñera, y el 75\% cuando el contrincante es Lavín, porcentajes superiores al apoyo hacia la candidata en primera vuelta ${ }^{14}$. De esta forma, existe cierta evidencia de que el apoyo DC a Bachelet fue fundamental para consagrar el cuarto gobierno de la Concertación.

${ }^{14}$ Otra interpretación sugiere que la ventaja de Bachelet en segunda vuelta se explica por la influencia del PC. De hecho, el coeficiente de correlación entre su votación y la de Bachelet en la segunda vuelta fue de $0,381(* * *)$, aunque inferior a la relación que en la misma instancia existió con Lagos, cuyo coeficiente fue de 0,434 $(* * *)$. 


\section{Conclusiones}

El PDC, siendo aún relevante en términos electorales y en su presencia en la Cámara y en el Senado, ha ido perdiendo apoyo ciudadano. A esto debemos sumar su menor impacto en las candidaturas presidenciales de la Concertación en los dos últimos comicios. Se evidenciarían, además, algunos problemas en la selección de candidatos, particularmente para las últimas elecciones, ya que parte importante de ellos no superó el 20\%, marcando grandes diferencias con los comicios de diputados anteriores.

Las variables socioeconómicas y sociodemográficas a nivel comunal, por sí solas, tienen una baja capacidad explicativa del desempeño electoral del PDC. Sin embargo, "religión” y "escolaridad” parecen determinar de mejor forma tanto el desempeño como la adhesión al partido. Particularmente para 1990, y con una muestra urbana, el hecho de ser católico se presentaba como un determinante claro a la hora de adherir al PDC en comparación con quienes no profesaban alguna religión, situación que se repite para el caso de los encuestados menos escolarizados al compararlos con los que tienen estudios superiores. Para 2005, y con una muestra nacional, la situación presenta ciertas modificaciones. La categoría "católico" deja de ser significativa y es más evidente la baja adhesión al PDC por parte de aquellos que profesan "otras religiones". Sin embargo, cuando acudimos a resultados de encuestas con muestras urbanas, particularmente las del ICSO-UDP, la filiación católica se presenta como un determinante robusto. Así, una posible interpretación es que dicho determinante mantiene vigencia en los sectores urbanos, mas no necesariamente en los rurales. Esto parece ser concordante con los resultados electorales por comuna, donde a mayor porcentaje de católicos, mayor votación por el PDC, más aún si consideramos que el peso relativo de las comunas urbanas es superior al de las rurales. Luego, por educación, la tendencia se mantiene tanto en las encuestas como en los resultados electorales por comunas. Las personas con menor nivel educativo presentan mayor disposición a votar por el PDC, lo que concuerda con los datos comunales: a mayor escolaridad promedio, menor votación por el PDC.

Finalmente concluimos que el PDC sigue siendo un partido de centro. La mayoría de sus adherentes se autoubica en este sector considerando la escala ideológica. A pesar de participar activamente en una coalición de centroizquierda, los adherentes al partido parecen defender el espacio natural que por décadas se le atribuyó. Esto, indudablemente, trae ciertas dificultades. Aunque no lo analizamos en este trabajo, el votante DC parece ser más permeable a las dinámicas de voto cruzado, particularmente en los co- 
micios de 2005 que, por su carácter concurrente, nos permiten estudiar este fenómeno de manera más certera. De esta forma, podríamos explicar por qué la candidata de la Concertación obtuvo menos votos que la lista parlamentaria del pacto, observando la menor disposición de los votantes DC para apoyar a los candidatos presidenciales que no eran militantes del partido. Esto se refleja tanto en los resultados de las encuestas como en los datos electorales por comuna.

\section{BiBLIOGRAFÍA}

Altman, David: "Redibujando el Mapa Electoral Chileno: Incidencia de Factores Socioeconómicos y Género en las Urnas”. En Revista de Ciencia Política, 24: (2), 2004, pp. 49-66.

Angell, Alan: "Party Change in Chile in Comparative Perspective". En Revista de Ciencia Política, 23: (2), 2003, pp. 88-108.

Arriagada, Genaro: De la Vía Chilena a la Vía Insurreccional. Santiago: Editorial del Pacífico, 1974.

Democracia Cristiana y Partido Comunista. Santiago: Aconcagua, 1986.

"El Resultado de las Elecciones 2001 y su Proyección Estratégica”. En Asuntos Públicos, 168, 2001. Artículo en línea, disponible en: http:// www.asuntospublicos.org/informe.php?id=168

“2005: La Situación Político-Electoral de la Democracia Cristiana”. En Asuntos Públicos, 515. 2005. Artículo en línea, disponible en: http:// www.asuntospublicos.org/informe.php?id=3064

Boeninger, Edgardo: Democracia en Chile. Lecciones para la Gobernabilidad. Santiago: Andrés Bello, 1997.

Cabezas, José Miguel y Patricio Navia: “El Efecto del Sistema Binominal en el Número de Candidatos y de Partidos en las Elecciones Legislativas en Chile, 19892001”. En Política, 45, 2005, pp. 29-52.

Cain, Bruce, John Ferejohn y Morris P. Fiorina: The Personal Vote. Cambridge: Harvard University Press, 1987.

Campbell, Angus, Philip Converse, Warren Miller y Donald Stokes: The American Voter. New York: John Wiley, 1960.

Campbell, Angus y Warren E. Miller: “The Motivational Basis of Straight and Split Ticket Voting”. En The American Political Science Review, 51 (2), 1957, pp. 293-312.

Cavallo, Ascanio: La Historia Oculta de la Transición. Santiago: Grijalbo, 1998.

Cavallo, Ascanio, Oscar Sepúlveda y Manuel Salazar: La Historia Oculta del Régimen Militar: 1973-1988. Santiago: Grijalbo, 1999.

Centro de Estudios Públicos: "Estudio Social y de Opinión Pública Nº 11. Segunda Serie, Diciembre 1990”. Santiago: Centro de Estudios Públicos.

— "Estudio Nacional de Opinión Pública N 10. Octubre 1999”. Santiago:

Centro de Estudios Públicos.

"Estudio Nacional de Opinión Pública No 23. Tercera Serie, Octubre Noviembre 2005”. Santiago: Centro de Estudios Públicos. 
Converse, Philip: “Of Time and Partisan Stability”. En Comparative Political Studies 2, 1969, pp. 139-171.

Crewe, Ivor: "Voters, Parties and Leaders Thirty Years On: Western Electoral Studies and the New Democracies of Eastern Europe”. En Ian Budge y David McKay (eds.), Developing Democracy. London: Sage Publications, 1995, pp. 53-78.

Dix, Robert: "Democratization and the Institutionalization of Latin American Political Parties”. En Comparative Political Studies, 24: (4), 1992, pp. 488-511.

Drake, Paul e Iván Jaksic: El Modelo Chileno. Democracia y Desarrollo en los 90. Santiago: LOM, 1999.

Duverger, Maurice: Los Partidos Políticos. México: Fondo de Cultura Económica, [1951] (2002).

Fiorina, Morris P.: Divided Government. New York: Macmillan, 1992.

Fleet, Michael: The Rise and Fall of Chilean Christian Democracy. Princeton: Princeton University Press, 1985.

Fogarty, Michael: Christian Democracy in Western Europe. 1820-1953. London: Routledge y Kegan, 1958.

Garretón, Manuel: "Partidos, Transición y Democracia”. Documento de Trabajo, $\mathrm{N}^{\circ}$ 443. Santiago: FLACSO, 1990.

Garrido, Carolina y Patricio Navia: "Candidatos Fuertes en la Concertación. ¿'Seguro para los Subcampeones o Prevalencia de los Dos Tercios?”. En Estudios Públicos, 99, 2005, pp. 165-194.

Hanley, David (ed.): Christian Democracy in Europe: a Comparative Perspective. London: New York Pinter, 1994.

Huneeus, Carlos: “La Vigencia de la DC”. En Asuntos Públicos, 186, 2002. Artículo en línea, disponible en: http://www.asuntospublicos.org/informe.php?id=186 “ ¿Dónde Se Fueron los Votantes del PDC?”. En Asuntos Públicos, 175, 2002a. Artículo en línea, disponible en: http://www.asuntospublicos.org/ informe.php?id=175

“A Highly Institutionalized Political Party: Christian Democracy in Chile”. En Scott Mainwaring y Timothy Scully (eds.), Christian Democracy in Latin America, Electoral Competition and Regime Conflicts. Stanford: Stanford University Press, 2003, pp 121-162.

INE (Instituto Nacional de Estadísticas): www.ine.cl

Jacobson, Gary: The Electoral Origins of Divided Government. Competition in US House Election 1946-1988. Boulder, Colorado: Westview Press, 1990.

Jenings, M. Kent y Richard Niemi: “The Transmission of Political Values from Parent to Child”. En American Political Science Review, LXII (1), 1968, pp. 169184.

Kalyvas, Stathis N.: The Rise of Christian Democracy in Europe. London: Cornell University Press, 1996.

Lazarsfeld, Paul, Bernard Berelson y Hazel Gaudet: The People's Choice. New York: Columbia University Press, 1944.

Lipset, S. M.: “The Indispensability of Political Parties”. En Journal of Democracy, 11: (1), 2000, pp. 48-55.

López, Miguel Ángel. "Conducta Electoral y Estratos Económicos: El Voto de los Sectores Populares en Chile”. En Política, 43, 2004, pp. 285-298. 
Magar, Erica, Marc Rosemblum, y David Samuels: "On the Absence of Centripetal Incentives in Double-Member Districts. The Case of Chile”. En Comparative Political Studies, 31 (6), 1998, pp. 714-739.

Mardones, Rodrigo: “Descentralización y Transición en Chile”. En Revista de Ciencia Política, 26: (1), 2006, pp. 3-24.

Montes, Esteban, Scott Mainwaring y Eugenio Ortega: "Rethinking the Chilean Party System”. En Journal of Latin American Studies, 32: (3), 2000, pp. 795-824.

Morlino, Leonardo: "Political Parties and Democratic Consolidation in Southern Europe”. En Richard Gunther, P. Nikiforos Diamandouros y Hans Jürgen Puhle (eds.), The Politics of Democratic Consolidation. Southern Europe in Comparative Perspective. Baltimore: The Johns Hopkins University Press, 1995, pp. 315-388.

Navarrete, Bernardo: Las Tendencias Electorales de los Partidos de Centro. El Caso de la Democracia Cristiana Chilena. Santiago: Fundación Konrad Adenauer, 2003.

"Un Centro Excéntrico. Cambio y Continuidad en la Democracia Cristiana 1957-2005”. En Política, 45, 2005, pp. 109-146.

Navia, Patricio: "Dónde Fueron los Votos del PDC”. Working Paper (documento de trabajo), Instituto de Estudios Políticos de la Universidad Nacional Andrés Bello, 2002.

"La Transformación de Votos en Escaños: Leyes Electorales en Chile, 18332004”. En Política y Gobierno, XII (2), 2005, pp. 233-276.

Niemi, Richard G. y Herbert F. Weisberg (eds.): Controversies in Voting Behavior. Washington, DC: CQ Press, 2001.

Nohlen, Dieter: Sistemas Electorales y Partidos Políticos. México: FCE, 1995.

Ortega Frei, Eugenio: Historia de una Alianza. El Partido Socialista de Chile y el Partido Demócrata Cristiano. 1973-1988. Santiago: CED-CESOC, 1992.

- "Los Partidos Políticos Chilenos: Cambio y Estabilidad en el Comportamiento Electoral 1990-2000”. En Revista de Ciencia Política, 23: (2), 2003, pp. 109-147.

Otano, Rafael: Crónica de la Transición. Santiago: Planeta, 1995.

Richardson, Bradley: “European Party Royalties Revisited”. En American Political Science Review, 85, 1991, pp. 751-775.

Rosenstone, Steven J. y John Mark Hansen: Mobilization, Participation, and Democracy in America. London: Longman, 2002.

Sartori, Giovanni: Partidos y Sistemas de Partidos. Madrid: Alianza, 1992.

Scully, Timothy: Los Partidos de Centro y la Evolución Política Chilena. Santiago: CIEPLAN, 1992.

Scully, Timothy y Samuel Valenzuela: "De la Democracia a la Democracia. Continuidad y Variaciones en las Preferencias del Electorado y en el Sistema de Partidos en Chile”. En Estudios Públicos, 5, 1993, pp. 195-228.

Siavelis, Peter: "Continuity and Change in the Chilean Party System”. En Comparative Political Studies, 30: (6), 1997, pp. 651-674.

Taagepera, Rein y Bernard Grofman: "Mapping the Indices of Seats-Votes Disproportionality and Inter-Election Volatility”. En Party Politics, 9 (6), 2003, pp. 659-677.

Tironi, Eugenio y Felipe Agüero: “Sobrevivirá el Nuevo Paisaje Político Chileno”. En Estudios Públicos, 74, 1999, pp. 151-168. 
Torcal, Mariano y Scott Mainwaring: "The Political Recrafting of Social Bases of Party Competition: Chile, 1973-95”. En British Journal of Political Science, 33: (1), 2003, pp. 55-84.

Valenzuela, Arturo: El Quiebre de la Democracia en Chile. Santiago: Colección Ciencia Política Universidad Diego Portales, 2003.

Valenzuela, Samuel: “Orígenes y Transformación del Sistema de Partidos en Chile”. En Estudios Públicos, 58, 1995, pp. 5-80.

- "Respuesta a Eugenio Tironi y Felipe Agüero: Reflexiones sobre el Presente y Futuro del Paisaje Político Chileno a la Luz de su Pasado”. En Estudios Públicos, 75, 1999, pp. 273-290.

Ventura, Raphael: "Family Political Socialization in Multiparty Systems”. En Comparative Political Studies, 34 (6), 2001, pp. 666-691.

Walker, Ignacio: El Futuro de la DC. Santiago: Ediciones B, 1999. 
